\title{
GLOBAL AND LOCAL ERROR ANALYSIS FOR THE RESIDUAL-FREE BUBBLES METHOD APPLIED TO ADVECTION-DOMINATED PROBLEMS.
}

\author{
GIANCARLO SANGALLI *
}

Abstract. We prove the stability and a priori global and local error analysis for the residual-free bubbles finite element method applied to advection dominated advection-diffusion problems.

Key words. advection-diffusion problems, local error estimates, residual free bubbles.

AMS subject classifications. 65G99, 65N30, 76R99

1. Introduction. We consider the linear partial differential equation problem

$$
\left\{\begin{array}{rlrl}
-\varepsilon \Delta u+\mathbf{c} \cdot \nabla u=f & & \text { in } \Omega \\
u & =0 & & \text { on } \partial \Omega,
\end{array}\right.
$$

where the unknown $u$ is a real-valued function on a convex polygonal domain $\Omega$ in $\mathbb{R}^{2}, \varepsilon$ is the strictly positive diffusion parameter and $\mathbf{c}$ is the velocity field on $\Omega$. This is a model problem in fluid dynamics and presents some of the difficulties that are encountered in the numerical simulations of fluid flows (see, for example, [20]). We suppose that $f$ belongs to $L^{2}(\Omega)$ and assume that $\mathbf{c}$ is continuously differentiable and verifies

$$
\left\{\begin{array}{l}
\operatorname{div}(\mathbf{c}) \leq 0 \quad \text { in } \Omega \\
\mathbf{c} \text { has no closed integral curves in } \bar{\Omega} .
\end{array}\right.
$$

Introducing the bilinear form

$$
a(w, v):=\varepsilon \int_{\Omega} \nabla w \nabla v d \mathbf{x}+\int_{\Omega}(\mathbf{c} \cdot \nabla w) v d \mathbf{x},
$$

and the linear functional

$$
\langle f, v\rangle:=\int_{\Omega} f v d \mathbf{x},
$$

problem (1.1) admits the variational formulation

$$
\left\{\begin{array}{l}
\text { Find } u \in H_{0}^{1}(\Omega) \text { such that } \\
a(u, v)=\langle f, v\rangle \quad \forall v \in H_{0}^{1}(\Omega) ;
\end{array}\right.
$$

there exists a unique solution $u$ of (1.4) and the following stability condition holds true (see for example [19]): there is a constant $C$ dependent only on $\Omega$ and $\mathbf{c}$ such that

$$
\varepsilon^{1 / 2}|u|_{H^{1}(\Omega)}+\|u\|_{L^{2}(\Omega)} \leq C\|f\|_{L^{2}(\Omega)} .
$$

*Dipartimento di Matematica "F. Casorati", Università di Pavia, Via Ferrata 1, 27100 Pavia, Italy (sangalli@dimat.unipv.it). 
It is well known that standard numerical methods (like central finite difference or standard Galerkin finite element methods) are inadequate when the quantity $\varepsilon /|\mathbf{c}|$ is small compared to the discretization step size, since the numerical solutions exhibit unphysical oscillatory behavior. In order to overcome this undesirable feature the so called stabilized methods introduce a proper modification of the variational formulation: given a triangulation $\mathcal{T}_{h}$ of the domain and the integer $k \geq 1$ we consider the usual finite dimensional space

$$
W_{h} \equiv W_{h}^{k}\left(\mathcal{T}_{h}, \Omega\right):=\left\{\begin{array}{c}
v \in H_{0}^{1}(\Omega): v \text { is polynomial of } \\
\text { degree } \leq k \text { on each triangle in } \mathcal{T}_{h}
\end{array}\right\}
$$

a generic stabilized method now reads

$$
\left\{\begin{array}{l}
\text { Find } u_{h}^{S} \in W_{h} \text { such that } \\
a\left(u_{h}^{S}, v_{h}\right)+S\left(u_{h}^{S}, v_{h}\right)=\left\langle f, v_{h}\right\rangle \quad \forall v_{h} \in W_{h},
\end{array}\right.
$$

where the form $S$ should provide a better stability behavior. This abstract framework (studied in [2]) includes the SUPG method proposed by Hughes and co-authors (in [9]), which corresponds to the choice

$$
S\left(u_{h}^{S}, v_{h}\right)=\sum_{T \in \mathcal{T}_{h}} \tau_{T} \int_{T}\left[f-\left(-\varepsilon \Delta u_{h}^{S}+\mathbf{c} \cdot \nabla u_{h}^{S}\right)\right]\left(-\varepsilon \Delta v_{h}-\mathbf{c} \cdot \nabla v_{h}\right) d \mathbf{x},
$$

where the artificial streamline diffusion coefficient $\tau_{T}$ is suitably chosen.

The residual-free bubble approach for problem (1.1), proposed by Brezzi and Russo in [8], is inspired by a different philosophy: taking the variational formulation (1.4) without modification, the numerical solution is found in a proper space $V_{h}$ richer than $W_{h}$. With the definition

$$
V_{h} \equiv V_{h}^{k}\left(\mathcal{T}_{h}, \Omega\right):=\left\{\begin{array}{c}
v \in H_{0}^{1}(\Omega): v \text { is polynomial of } \\
\text { degree } \leq k \text { on each edge of } T \text { in } \mathcal{T}_{h}
\end{array}\right\},
$$

the RFB (Residual-free Bubbles) method simply reads

$$
\left\{\begin{array}{l}
\text { Find } u_{h} \in V_{h} \text { such that } \\
a\left(u_{h}, v_{h}\right)=\left\langle f, v_{h}\right\rangle \quad \forall v_{h} \in V_{h} .
\end{array}\right.
$$

Actually it is possible to compute numerically an accurate approximation of the solution $u_{h}$ of problem (1.10), even if it is an infinite dimensional problem. The interested reader may see [8] and [5], where the numerical procedure is proposed and tested (with $k=1$ and triangular or quadrilateral elements), or [14] and [6] for the general procedure. We note that in the case $k=1$ (with triangular elements and piecewise constant data) the RFB and SUPG methods are related: indeed the piecewise linear nodal interpolant of the RFB solution verifies the SUPG variational formulation (1.7)-(1.8) with a proper choice of $\tau_{T}$ (see [8]). So, in this case, the RFB approach can be considered a justification of the SUPG stabilization as well as a way of choosing $\tau_{T}$. A deeper investigation of the relative merits and drawbacks of the two approaches would be very interesting, but goes beyond the scopes of this paper.

The aim of this paper is the error analysis for the RFB method. In the case $k=1$ we can refer to the SUPG error analysis, as a consequence of the relation mentioned 
before (see also [7]). The general case has already been considered in [6], where error estimates are obtained on the whole domain $\Omega$ with respect to the so called energy norm (namely, in this case, $\varepsilon^{1 / 2}|\cdot| H_{H^{1}(\Omega)}$ ). In the present work we obtain:

1. a convergence result in a proper negative order norm, without any extra regularity assumption on the exact solution $u$;

2. a stability estimate and a global error estimate in the $L^{2}$-norm;

3. a local error estimate.

It is well known that, when $\varepsilon$ approaches zero, the solution $u$ of problem (1.1) exhibits layers, i.e. narrow regions where $u$ changes very rapidly. This fact causes the global error estimates to be useless in practice. The fundamental result of this paper, the local error analysis, guarantees that the RFB method works fine also in the presence of layers.

The same error estimates for the SUPG method have been proven by Johnson and co-authors in the eighties (see [17], [18] and [19]). In this work we follow a similar technique, although we use extensively some properties of the space $V_{h}$ which are investigated in section 2 . We assume there that when $k \geq 2$, the angle between the triangle's edges and the field $\mathbf{c}$ is uniformly bounded away from zero: it is a technical but restrictive hypothesis. Similar hypotheses are sometimes assumed in the RFB stability analysis (see for example [13]); the possibility of dropping them could be the subject of a further work. Sections 3 and 4 are devoted to the global and local analysis respectively.

2. Statement of the problem and properties of the functions of $V_{h}$. In the following we shall denote by $D_{\mathbf{v}}^{i}$ the $i$-th order derivative in the direction of $\mathbf{v} \in \mathbb{R}^{2}$ and by $C$ and $C_{i}$ (where $i$ is an integer index) positive constants independent on $f$, $u, u_{h}, \varepsilon$ and $h$, but whose value may change at each occurrence. We shall use the standard notation to refer to Hilbertian Sobolev spaces, norms and seminorms (as, for example, in [10] and [16]). For the sake of simplicity we shall use the same notation to refer both to a function or to its restriction to a subset of the original domain.

We assume to deal with a regular and quasi-uniform family of triangulations $\mathcal{T} \equiv\left\{\mathcal{T}_{h}\right\}_{h \in \mathcal{H}}$, where $\mathcal{H}$ is a set of positive indices that have the meaning of meshsize, as it will be made precise in the sequel; $\mathcal{T}_{h}$ is a set of disjoint open triangles such that

$$
\bigcup_{T \in \mathcal{T}_{h}} \bar{T}=\bar{\Omega}
$$

with the assumption that each vertex of each triangle is a vertex of adjacent triangles and that there exist constants $C_{1}=C_{1}(\mathcal{T})$ and $C_{2}=C_{2}(\mathcal{T})$ such that

$$
C_{1} h_{T} \leq h \leq C_{2} \rho_{T} \quad \forall T \in \mathcal{T}_{h},
$$

where $h_{T}$ is the diameter of $T$ and $\rho_{T}$ is the radius of the biggest circle belonging to $\bar{T}$.

We restrict ourselves to the advection-dominated case, assuming that

$$
\mathbf{c}(\mathbf{x}) \neq \mathbf{0} \quad \forall \mathbf{x} \in \bar{\Omega},
$$

and

$$
\varepsilon \leq h \leq 1
$$

Our last assumption relates the triangulation family $\mathcal{T}$ to the advection field $\mathbf{c}$. If we consider $k \geq 2$ then we assume that there exists a positive constant $C=C(\mathcal{T}, \mathbf{c})$ 
such that

$$
|\mathbf{c} \cdot \mathbf{n}|>C \text { on } \partial T \quad \forall h \in \mathcal{H}, \forall T \in \mathcal{T}_{h},
$$

where $\mathbf{n}$ denotes the outward normal vector defined on $\partial T$ (in the notation the dependence on $T$ is neglected). In the case $k=1$ we make the weaker hypothesis that there exists a positive constant $C=C(\mathcal{T}, \mathbf{c})$ such that

$$
|\mathbf{c} \cdot \mathbf{n}|>C \text { on at least two of the three edges of } T \quad \forall h \in \mathcal{H}, \forall T \in \mathcal{T}_{h} .
$$

The hypothesis (2.4) is of course restrictive, since e.g. triangles whose edges are aligned with the field $\mathbf{c}$ are not allowed. In contrast with this, the weaker hypothesis (2.5) (that we assume in the case $k=1$ ) is not very restrictive: indeed it may be proved that (2.5) is fulfilled for $h$ sufficiently small since $\mathbf{c}$ is regular and the edges of any triangle, by virtue of (2.1), form angles uniformly bounded away from zero.

Now we recall some standard definitions and results that we shall use in the sequel. We define the reference triangle $\widehat{T}$ as

$$
\widehat{T}=\left\{\left(\widehat{x}_{1}, \widehat{x}_{2}\right) \in \mathbb{R}^{2}: 0<\widehat{x}_{1}<1,0<\widehat{x}_{2}<1-\widehat{x}_{1}\right\},
$$

and we denote the three edges of the triangle as

$$
\begin{aligned}
& \partial \widehat{T}_{1}=\left\{\left(\widehat{x}_{1}, \widehat{x}_{2}\right) \in \mathbb{R}^{2}: \widehat{x}_{1}=0,0<\widehat{x}_{2}<1\right\}, \\
& \partial \widehat{T}_{2}=\left\{\left(\widehat{x}_{1}, \widehat{x}_{2}\right) \in \mathbb{R}^{2}: 0<\widehat{x}_{1}<1, \widehat{x}_{2}=0\right\}, \\
& \partial \widehat{T}_{3}=\left\{\left(\widehat{x}_{1}, \widehat{x}_{2}\right) \in \mathbb{R}^{2}: 0<\widehat{x}_{1}<1, \widehat{x}_{2}=1-\widehat{x}_{1}\right\} .
\end{aligned}
$$

We can associate with each triangle $T$ in $\mathcal{T}_{h}$ an affine bijective map $B_{T}: \widehat{T} \rightarrow T$, and consequently we enumerate the edges on each $T$ in such a way that the notations agree:

$$
\partial T_{i}=B_{T}\left(\partial \widehat{T}_{i}\right) \quad i=1,2,3 .
$$

By virtue of $(2.1)$, there exists a constant $C=C(\mathcal{T})$ such that for each $T$ in $\mathcal{T}_{h}$

$$
\left\|B_{T}\right\| \leq C h \text { and }\left\|B_{T}^{-1}\right\| \leq C h^{-1} .
$$

Given a function $v: T \rightarrow \mathbb{R}$ we define as usual the pull-back $\widehat{v}: \widehat{T} \rightarrow \mathbb{R}$ as

$$
\widehat{v}=v \circ B_{T}
$$

in this case $v$ and $\widehat{v}$ verify the so called scaling inequalities (where $H^{0}$ simply denotes the space $L^{2}$ ): there exist constants $C_{1}=C_{1}(\mathcal{T})$ and $C_{2}=C_{2}(\mathcal{T})$ such that for each $T \in \mathcal{T}_{h}$ and for all $r \geq 0$

$$
C_{1} h^{1-r}|\widehat{v}|_{H^{r}(\widehat{T})} \leq|v|_{H^{r}(T)} \leq C_{2} h^{1-r}|\widehat{v}|_{H^{r}(\widehat{T})} \quad \forall v \in H^{r}(T) ;
$$

analogously on the boundary of the triangles we have for every $r$ such that $0 \leq r \leq 1$

$$
C_{1} h^{1 / 2-r}|\widehat{v}|_{H^{r}(\partial \widehat{T})} \leq|v|_{H^{r}(\partial T)} \leq C_{2} h^{1 / 2-r}|\widehat{v}|_{H^{r}(\partial \widehat{T})} \quad \forall v \in H^{r}(\partial T) .
$$

Since the boundary $\partial T$ of the triangles is a manifold with corners, spaces of more regular functions are not well defined on $\partial T$ (see [16]). Otherwise on each edge $\partial T_{i}$ 
we can define any Sobolev space and in this case, for each triangle $T$, each $r>0$, and $i=1,2,3$ we have:

$$
C_{1} h^{1 / 2-r}|\widehat{v}|_{H^{r}\left(\partial \widehat{T}_{i}\right)} \leq|v|_{H^{r}\left(\partial T_{i}\right)} \leq C_{2} h^{1 / 2-r}|\widehat{v}|_{H^{r}\left(\partial \widehat{T}_{i}\right)} \quad \forall v \in H^{r}\left(\partial T_{i}\right) .
$$

We recall that for functions belonging to $V_{h}$, which are polynomials on each edge of triangles in $\mathcal{T}_{h}$, we have the so called inverse inequalities: there exists a constant $C=C(\mathcal{T})$ such that

$$
\left\{\begin{array}{l}
\forall T \in \mathcal{T}_{h}, \forall v_{h} \in V_{h}, \forall r: 0 \leq r \leq 1 \\
\left|v_{h}\right|_{H^{r}(\partial T)} \leq C h^{-r}\left\|v_{h}\right\|_{L^{2}(\partial T)}
\end{array}\right.
$$

and

$$
\left\{\begin{array}{l}
\forall T \in \mathcal{T}_{h}, \forall v_{h} \in V_{h}, \forall r \geq 0 \text { and } i=1,2,3 \\
\left|v_{h}\right|_{H^{r}\left(\partial T_{i}\right)} \leq C h^{-r}\left\|v_{h}\right\|_{L^{2}\left(\partial T_{i}\right)} .
\end{array}\right.
$$

The following known result extends the standard lifting theorem for traces.

LEMma 2.1. Given a function $\widehat{w}_{0} \in H^{1 / 2}(\partial \widehat{T})$ and a real parameter $t$ with $0<t \leq 1$, there exists $\widehat{w} \in H^{1}(\widehat{T})$ such that $\widehat{w}=\widehat{w}_{0}$ on $\partial \widehat{T}$ and

$$
t|\widehat{w}|_{H^{1}(\widehat{T})}^{2}+t^{-1}\|\widehat{w}\|_{L^{2}(\widehat{T})}^{2} \leq C\left(t\left|\widehat{w}_{0}\right|_{H^{1 / 2}(\partial \widehat{T})}^{2}+\left\|\widehat{w}_{0}\right\|_{L^{2}(\partial \widehat{T})}^{2}\right),
$$

where $C$ does not depend on $t$ and $\widehat{w}_{0}$.

Proof. The function $\widehat{w}$ minimizing the left hand side of (2.11), among those admitting the trace $\widehat{w}_{0}$, is the solution of the elliptic b.v.p.

$$
\left\{\begin{aligned}
-t \Delta \widehat{w}+t^{-1} \widehat{w} & =0 & & \text { in } \widehat{T} \\
\widehat{w} & =\widehat{w}_{0} & & \text { in } \partial \widehat{T} .
\end{aligned}\right.
$$

Then we can obtain (2.11) using the usual properties of the elliptic problem (2.12) (see for example [16] where it is considered the case of domains with polygonal boundaries). We refer to [1] for details, where a similar result is proved.

REMARK 2.2. Note that we can state a converse of Lemma 2.1 (see Theorem 1.5.1.3 and 1.5.1.10 in [16]): there exists a positive constant $C$ such that each function $\widehat{v}$ in $H^{1}(\widehat{T})$ verifies

$$
t|\widehat{v}|_{H^{1 / 2}(\partial \widehat{T})}^{2}+\|\widehat{v}\|_{L^{2}(\partial \widehat{T})}^{2} \leq C\left(t|\widehat{v}|_{H^{1}(\widehat{T})}^{2}+t^{-1}\|\widehat{v}\|_{L^{2}(\widehat{T})}^{2}\right) \quad \forall t: 0<t \leq 1 .
$$

Corollary 2.3. Assume that (2.1) and (2.3) hold true. Then for each $T \in \mathcal{T}_{h}$ and each $w_{0} \in H^{1 / 2}(\partial T)$, there exists a function $w \in H^{1}(T)$ with $w=w_{0}$ on $\partial T$ and

$$
\varepsilon|w|_{H^{1}(T)}^{2}+\varepsilon^{-1}\|w\|_{L^{2}(T)}^{2} \leq C\left(\varepsilon\left|w_{0}\right|_{H^{1 / 2}(\partial T)}^{2}+\left\|w_{0}\right\|_{L^{2}(\partial T)}^{2}\right),
$$

where $C$ depends only on $\mathcal{T}$.

Proof. Following a standard argument, we apply Lemma 2.1 with $t=\epsilon h^{-1}$ and $\widehat{w}_{0}=w_{0} \circ B_{T}$. Using the scaling properties (2.6) and (2.7) we get (2.13). 
Corollary 2.4. Assume that (2.1) and (2.3) hold true. Then for each $T \in \mathcal{T}_{h}$, each $w_{0} \in H^{1 / 2}(\partial T)$ and each $v \in H^{1}(T)$ there exists a function $w \in H^{1}(T)$ with $w=w_{0}$ on $\partial T$ and verifying

$$
\begin{aligned}
& \varepsilon|v-w|_{H^{1}(T)}^{2}+\varepsilon^{-1}\|v-w\|_{L^{2}(T)}^{2} \\
& \quad \leq C\left(\varepsilon\left|v-w_{0}\right|_{H^{1 / 2}(\partial T)}^{2}+\left\|v-w_{0}\right\|_{L^{2}(\partial T)}^{2}\right),
\end{aligned}
$$

where $C$ depends only on $\mathcal{T}$.

Proof. This is a direct application of the previous corollary to the function $v-w_{0}$ defined on $\partial T$.

We can now discuss the crucial point of our analysis: the approximation in $V_{h}$ of a generic function. Remember that the functions of $V_{h}$ are piecewise polynomial on the boundary of the triangles. Then, given a function $v$ on $\Omega$, it seems natural to define on each $\partial T$ its approximation $\Pi_{h, \varepsilon}(v)$ as the usual nodal interpolant of the trace of $v$ on $\partial T$. Assuming this trace regular enough (as we shall make precise below) and using (2.9) and (2.10), we have the error estimate

$$
\begin{aligned}
& h^{1 / 2}\left|v-\Pi_{h, \varepsilon}(v)\right|_{H^{1 / 2}(\partial T)}+\left\|v-\Pi_{h, \varepsilon}(v)\right\|_{L^{2}(\partial T)} \\
& \leq C \sum_{i=1}^{3} h\left|v-\Pi_{h, \varepsilon}(v)\right|_{H^{1}\left(\partial T_{i}\right)}+\left\|v-\Pi_{h, \varepsilon}(v)\right\|_{L^{2}\left(\partial T_{i}\right)} \\
& \quad \leq C h^{k+1} \sum_{i=1}^{3}|v|_{H^{k+1}\left(\partial T_{i}\right)} .
\end{aligned}
$$

In order to extend the definition of $\Pi_{h, \varepsilon}(v)$ in the interior of the triangles we follow triangle by triangle the procedure of Corollary 2.4, i.e. we define $\Pi_{h, \varepsilon}(v)$ on $T$ as the function $w \in H^{1}(T)$ corresponding to $w_{0}=\Pi_{h, \varepsilon}(v) \in H^{1 / 2}(\partial T)$, as in Corollary 2.4. The accuracy inside any triangle depends on the accuracy on its boundary, in agreement with (2.14). In conclusion, assuming (2.3), for any $v \in H_{0}^{1}(\Omega) \cap C(\partial T)$ with $v \in H^{k+1}\left(\partial T_{i}\right), i=1,2,3$, we have the local error estimate

$$
\begin{aligned}
& \varepsilon^{1 / 2} \mid v-\left.\Pi_{h, \varepsilon}(v)\right|_{H^{1}(T)}+\varepsilon^{-1 / 2}\left\|v-\Pi_{h, \varepsilon}(v)\right\|_{L^{2}(T)} \\
& \leq C h^{k+1} \sum_{i=1}^{3}|v|_{H^{k+1}\left(\partial T_{i}\right)},
\end{aligned}
$$

where $C$ depends only on $\mathcal{T}$ and $k$. Note that in (2.15) the accuracy of the approximation depends only on the regularity of $v$ on the boundary of the triangle: our Lemmas 3.2 and 4.3 are based on that. Otherwise there are some difficulties in the definition of $\Pi_{h, \varepsilon}(v)$ when the trace of $v$ is not regular enough. Then we define a new approximation, denoted as $P_{h, \varepsilon}(v)$, which coincides with the restriction of the quasi-interpolant of $v$ on any boundary of the triangles (see, for example, [21] for the definitions and properties of quasi-interpolant operators). This kind of approximation verifies

$$
h^{1 / 2}\left|v-P_{h, \varepsilon}(v)\right|_{H^{1 / 2}(\partial T)}+\left\|v-P_{h, \varepsilon}(v)\right\|_{L^{2}(\partial T)} \leq C h^{r-1 / 2}|v|_{H^{r}(\mathcal{N}(T))},
$$

where $\mathcal{N}(T)$ denotes the union of the triangles which have at least a vertex in common with $T, v$ is supposed regular enough and $r$ is a positive real number smaller than 
$k+1$. The extension of $P_{h, \varepsilon}(v)$ to the whole domain $\Omega$ is completed by using Corollary 2.4 , as in the previous case. Finally, for any function $v \in H_{0}^{1}(\Omega) \cap H^{r}(\mathcal{N}(T))$, we have

$$
\begin{aligned}
& \varepsilon^{1 / 2}\left|v-P_{h, \varepsilon}(v)\right|_{H^{1}(T)}+\varepsilon^{-1 / 2}\left\|v-P_{h, \varepsilon}(v)\right\|_{L^{2}(T)} \\
& \leq C h^{r-1 / 2}|v|_{H^{r}(\mathcal{N}(T))} .
\end{aligned}
$$

In order to clarify the use of estimate (2.16) in the error analysis we present now a first result, similar to the one proved in [6] with a different technique. Note that we do not make use of hypotheses (2.4) or (2.5) in the proof. Recall the so called Galerkin property

$$
a\left(u-u_{h}, v_{h}\right)=0 \quad \forall v_{h} \in V_{h} ;
$$

we define

$$
\begin{aligned}
e & :=P_{h, \varepsilon}(u)-u_{h}, \\
\eta & :=u-P_{h, \varepsilon}(u)
\end{aligned}
$$

so $(2.17)$ gives

$$
a\left(e, v_{h}\right)=-a\left(\eta, v_{h}\right) \quad \forall v_{h} \in V_{h} .
$$

Theorem 2.5. Assume that (1.2), (2.1) and (2.3) hold true. Let $u$ and $u_{h}$ be the solutions of (1.4) and (1.10) respectively, and suppose that $u \in H^{r}(\Omega)$ with $1 \leq r \leq k+1$. Then there exists a constant $C=C(\mathcal{T}, \mathbf{c}, k)$ such that

$$
\varepsilon^{1 / 2}\left|u-u_{h}\right|_{H^{1}(\Omega)}+h^{-1 / 2}\left\|\mathbf{c} \cdot \nabla\left(u-u_{h}\right)\right\|_{H^{-1}(\Omega)} \leq C h^{r-1 / 2}|u|_{H^{r}(\Omega)} .
$$

Proof. In the proof we assume that each constant $C$ is dependent on $\mathcal{T}$, $\mathbf{c}$ and $k$ at most.

In the first part of this proof we use a standard argument in the FEM error analysis. Integrating by parts we get

$$
\begin{aligned}
a\left(u-u_{h}, u-u_{h}\right) & =\varepsilon \int_{\Omega}\left|\nabla\left(u-u_{h}\right)\right|^{2} d \mathbf{x}+\int_{\Omega} \mathbf{c} \cdot \nabla\left(\frac{\left(u-u_{h}\right)^{2}}{2}\right) d \mathbf{x} \\
& =\varepsilon \int_{\Omega}\left|\nabla\left(u-u_{h}\right)\right|^{2} d \mathbf{x}-\int_{\Omega} \operatorname{div}(\mathbf{c}) \frac{\left(u-u_{h}\right)^{2}}{2} d \mathbf{x} ;
\end{aligned}
$$

since the last term is non-negative, by assumption (1.2), we have the coercivity inequality

$$
a\left(u-u_{h}, u-u_{h}\right) \geq \varepsilon\left|u-u_{h}\right|_{H^{1}(\Omega)}^{2} .
$$

Moreover from (2.17) we have

$$
a\left(u-u_{h}, e\right)=0,
$$

that is

$$
a\left(u-u_{h}, u-u_{h}\right)=a\left(u-u_{h}, \eta\right) .
$$


Note that from (2.16), after squaring and summing over all triangles, we have

$$
\varepsilon^{1 / 2}|\eta|_{H^{1}(\Omega)}+\varepsilon^{-1 / 2}\|\eta\|_{L^{2}(\Omega)} \leq C h^{r-1 / 2}|u|_{H^{r}(\Omega)} .
$$

Then using the estimate (2.23) we get

$$
\begin{aligned}
a\left(u-u_{h}, \eta\right) & =\varepsilon \int_{\Omega} \nabla\left(u-u_{h}\right) \cdot \nabla \eta d \mathbf{x}+\int_{\Omega} \mathbf{c} \cdot \nabla\left(u-u_{h}\right) \eta d \mathbf{x} \\
& \leq C\left(\varepsilon^{1 / 2}\left|u-u_{h}\right|_{H^{1}(\Omega)}\right)\left(\varepsilon^{1 / 2}|\eta|_{H^{1}(\Omega)}+\varepsilon^{-1 / 2}\|\eta\|_{L^{2}(\Omega)}\right) \\
& \leq C\left(\varepsilon^{1 / 2}\left|u-u_{h}\right|_{H^{1}(\Omega)}\right)\left(h^{r-1 / 2}|u|_{H^{r}(\Omega)}\right)
\end{aligned}
$$

this last inequality with the coercivity condition (2.22) gives

$$
\varepsilon\left|u-u_{h}\right|_{H^{1}(\Omega)} \leq C h^{r-1 / 2}|u|_{H^{r}(\Omega)} .
$$

In the second part of the proof we estimate the streamline derivative of the error: for each $v \in H_{0}^{1}(\Omega)$ we set

$$
\begin{aligned}
\int_{\Omega} \mathbf{c} \cdot \nabla\left(u-u_{h}\right) v d \mathbf{x}= & \int_{\Omega} \mathbf{c} \cdot \nabla\left(u-u_{h}\right)\left(v-P_{h, \varepsilon}(v)\right) d \mathbf{x} \\
& +\int_{\Omega} \mathbf{c} \cdot \nabla\left(u-u_{h}\right) P_{h, \varepsilon}(v) d \mathbf{x} \\
= & I+I I ;
\end{aligned}
$$

on the first term we have, using (2.16) as before

$$
\begin{aligned}
I & \leq C\left|u-u_{h}\right|_{H^{1}(\Omega)}\left\|v-P_{h, \varepsilon}(v)\right\|_{L^{2}(\Omega)} \\
& \leq C\left(\varepsilon^{1 / 2}\left|u-u_{h}\right|_{H^{1}(\Omega)}\right)\left(\varepsilon^{-1 / 2}\left\|v-P_{h, \varepsilon}(v)\right\|_{L^{2}(\Omega)}\right) \\
& \leq C\left(\varepsilon^{1 / 2}\left|u-u_{h}\right|_{H^{1}(\Omega)}\right)\left(h^{1 / 2}|v|_{H^{1}(\Omega)}\right) ;
\end{aligned}
$$

using (2.16) and (2.17) we get

$$
\begin{aligned}
I I & =-\varepsilon \int \nabla\left(u-u_{h}\right) \cdot \nabla\left(P_{h, \varepsilon}(v)\right) d \mathbf{x} \\
& \leq\left(\varepsilon^{1 / 2}\left|u-u_{h}\right|_{H^{1}(\Omega)}\right)\left(\varepsilon^{1 / 2}\left|P_{h, \varepsilon}(v)\right|_{H^{1}(\Omega)}\right) \\
& \leq\left(\varepsilon^{1 / 2}\left|u-u_{h}\right|_{H^{1}(\Omega)}\right)\left(\varepsilon^{1 / 2}|v|_{H^{1}(\Omega)}+\varepsilon^{1 / 2}\left|v-P_{h, \varepsilon}(v)\right|_{H^{1}(\Omega)}\right) \\
& \leq C\left(\varepsilon^{1 / 2}\left|u-u_{h}\right|_{H^{1}(\Omega)}\right)\left(\varepsilon^{1 / 2}|v|_{H^{1}(\Omega)}+h^{1 / 2}|v|_{H^{1}(\Omega)}\right) .
\end{aligned}
$$

Finally

$$
\int_{\Omega} \mathbf{c} \cdot \nabla\left(u-u_{h}\right) v d \mathbf{x} \leq C\left(\varepsilon^{1 / 2}\left|u-u_{h}\right|_{H^{1}(\Omega)}\right)\left(h^{1 / 2}|v|_{H^{1}(\Omega)}\right),
$$

that is

$$
h^{-1 / 2}\left\|\mathbf{c} \cdot \nabla\left(u-u_{h}\right)\right\|_{H^{-1}(\Omega)} \leq C \varepsilon^{1 / 2}\left|u-u_{h}\right|_{H^{1}(\Omega)},
$$


and this concludes the proof.

REMARK 2.6. It is easy to see that

$$
\|\mathbf{c} \cdot \nabla v\|_{H^{-1}(\Omega)} \leq C\|v\|_{L^{2}(\Omega)} \quad \forall v \in L^{2}(\Omega),
$$

and that the two norms in (2.25) are not equivalent. The main reason is that the norm in the left hand side takes into consideration only the derivative in the direction of $\mathbf{c}$. More precisely $\|\nabla \cdot\|_{H^{-1}(\Omega)}$ and $\|\cdot\|_{L^{2}(\Omega)}$ are equivalent on the subset of functions having null mean value (see e.g. [11]). The error analysis with respect to the $L^{2}$-norm (with a lower order of convergence) is postponed to Theorem 3.5.

The following Proposition presents a key property of $V_{h}$ for our analysis. Note that this is exactly the point where we need the hypotheses (2.4) or (2.5) on the triangulation.

Proposition 2.7. Assume that (1.2), (2.1), (2.2), (2.3) and (2.4) (or (2.5) in the case $k=1$ ) hold true. Let $u_{h}$ be as in definition (1.10) and let $T$ be a triangle in $\mathcal{T}_{h}$. Then there exists a constant $C=C(\mathcal{T}, \mathbf{c})$ such that

$$
\left\|u_{h}\right\|_{L^{2}(\partial T)}^{2} \leq C\left(h^{-1}\left\|u_{h}\right\|_{L^{2}(T)}^{2}+\varepsilon\left|u_{h}\right|_{H^{1}(T)}^{2}+h\|f\|_{L^{2}(T)}^{2}\right) .
$$

Moreover let $u$ be as in definition (1.4) and e be as in (2.18); if $u \in H^{r}(\mathcal{N}(T))$ with $1 \leq r \leq k+1$ we have

$$
\|e\|_{L^{2}(\partial T)}^{2} \leq C\left(h^{-1}\|e\|_{L^{2}(T)}^{2}+\varepsilon|e|_{H^{1}(T)}^{2}+h^{2 r-1}|u|_{H^{r}(\mathcal{N}(T))}^{2}\right) .
$$

Proof. In the following proof we shall assume that the positive constants $C$ and $C_{i}$ are dependent only on $\mathcal{T}$, $\mathbf{c}$, and $k$ (and consequently on the constants appearing in $(2.4)$ or $(2.5))$.

In the first part of the proof we introduce a norm on $\partial T$ and we prove the equivalence with the norm $\|\cdot\|_{L^{2}(\partial T)}$. More precisely, we construct a weight function $\omega: \Omega \rightarrow \mathbb{R}$ such that

$$
\begin{array}{cc}
\omega \leq C & \text { on } \Omega, \\
\nabla \omega \leq C h^{-1} & \text { on } \Omega,
\end{array}
$$

and

$$
C_{1}\left\|v_{h}\right\|_{L^{2}(\partial T)}^{2} \leq \int_{\partial T} \omega v_{h}^{2} \mathbf{c} \cdot \mathbf{n} d \mathbf{x} \leq C_{2}\left\|v_{h}\right\|_{L^{2}(\partial T)}^{2} \quad \forall T \in \mathcal{T}_{h}, \forall v_{h} \in V_{h} .
$$

For each $i=1,2,3$ we define the functions $b_{i, T}: \bar{T} \rightarrow \mathbb{R}$ as

$$
b_{i, T}:=\prod_{\substack{j=1 \\ j \neq i}}^{3} \lambda_{j, T} \quad \text { on } \bar{T}
$$

where $\lambda_{j, T}$ denotes the usual $j$-th barycentric coordinate in $T$, whose value is 0 on $\partial T_{j}$ and 1 on the opposite vertex.

If we assume that (2.4) is fulfilled then $\mathbf{c} \cdot \mathbf{n}$ has constant sign on each $\partial T_{i}$; so we define the constants $s_{i}=\operatorname{sign}(\mathbf{c} \cdot \mathbf{n})_{\mid \partial T_{i}}$ and the function $\omega: \Omega \rightarrow \mathbb{R}$

$$
\omega:=\sum_{i=1}^{3} s_{i} b_{i, T} \quad \text { in } \bar{T}, \forall T \in \mathcal{T}_{h}
$$


the scaling inequalities (2.6) and (2.7), and the equivalence of norms on a finite dimensional space yield (2.28), (2.29) and (2.30).

Assume now that (2.5) holds true, that is, for example, $|\mathbf{c} \cdot \mathbf{n}|>C$ on $\partial T_{1}$ and $\partial T_{2}$. We define in this case

$$
\omega:=\sum_{i=1}^{2} s_{i} b_{i, T} \quad \text { in } \bar{T}, \forall T \in \mathcal{T}_{h} .
$$

Then we get (2.28) and (2.29) but, instead of (2.30), we can only infer

$$
C_{1}\left\|v_{h}\right\|_{L^{2}\left(\partial T_{1} \cup \partial T_{2}\right)}^{2} \leq \int_{\partial T} \omega v_{h}^{2} \mathbf{c} \cdot \mathbf{n} d \mathbf{x} \leq C_{2}\left\|v_{h}\right\|_{L^{2}\left(\partial T_{1} \cup \partial T_{2}\right)}^{2} \quad \forall v_{h} \in V_{h} ;
$$

but for piecewise linear functions on $\partial T$ (remember that (2.5) is restricted to the case $k=1$ ) we get the equivalence

$$
C_{1}\left\|v_{h}\right\|_{L^{2}\left(\partial T_{1} \cup \partial T_{2}\right)}^{2} \leq\left\|v_{h}\right\|_{L^{2}(\partial T)}^{2} \leq C_{2}\left\|v_{h}\right\|_{L^{2}\left(\partial T_{1} \cup \partial T_{2}\right)}^{2} \quad \forall v_{h} \in V_{h}, k=1,
$$

and (2.30) follows.

We now prove (2.26): using Gauss-Green formula we obtain

$$
\begin{aligned}
\int_{\partial T} \mathbf{c} \cdot \mathbf{n} u_{h}^{2} \omega d \sigma(\mathbf{x}) & =\int_{T} \operatorname{div}\left(\mathbf{c} u_{h}^{2} \omega\right) d \mathbf{x} \\
& =\int_{T} \operatorname{div}(\mathbf{c}) u_{h}^{2} \omega d \mathbf{x}+\int_{T} u_{h}^{2} \mathbf{c} \cdot \nabla \omega d \mathbf{x}+\int_{T} \mathbf{c} \cdot \nabla\left(u_{h}^{2}\right) \omega d \mathbf{x} \\
& =I+I I+I I I,
\end{aligned}
$$

and so, from (2.28) and (2.29) we obtain

$$
I+I I \leq C h^{-1}\left\|u_{h}\right\|_{L^{2}(T)}^{2} .
$$

Using Corollary 2.3 and the inverse estimate (2.9) we obtain a function $z_{h}$ such that $z_{h}=u_{h}$ on $\partial T$ and

$$
\varepsilon^{1 / 2}\left|z_{h}\right|_{H^{1}(T)}+\varepsilon^{-1 / 2}\left\|z_{h}\right\|_{L^{2}(T)} \leq C\left\|u_{h}\right\|_{L^{2}(\partial T)} .
$$

Since $\omega u_{h}-\omega z_{h}$ vanishes on $\partial T$ we have from (1.10)

$$
\varepsilon \int_{T} \nabla u_{h} \cdot \nabla\left(\omega u_{h}-\omega z_{h}\right) d \mathbf{x}+\int_{T} \mathbf{c} \cdot \nabla u_{h}\left(\omega u_{h}-\omega z_{h}\right) d \mathbf{x}=\int_{T} f\left(\omega u_{h}-\omega z_{h}\right) d \mathbf{x}
$$

reordering the terms:

$$
\begin{aligned}
\int_{T}\left(\mathbf{c} \cdot \nabla u_{h}\right) u_{h} \omega d \mathbf{x}= & -\varepsilon \int_{T} \nabla u_{h} \cdot \nabla u_{h} \omega d \mathbf{x}-\varepsilon \int_{T} u_{h} \nabla u_{h} \cdot \nabla \omega d \mathbf{x} \\
& +\varepsilon \int_{T} \nabla u_{h} \cdot \nabla z_{h} \omega d \mathbf{x}+\varepsilon \int_{T} z_{h} \nabla u_{h} \cdot \nabla \omega d \mathbf{x} \\
& +\int_{T}\left(\mathbf{c} \cdot \nabla u_{h}\right) z_{h} \omega d \mathbf{x} \\
& +\int_{T} f u_{h} \omega d \mathbf{x}-\int_{T} f z_{h} \omega d \mathbf{x} .
\end{aligned}
$$


So, using (2.3), (2.28) and (2.29) again we get

$$
\begin{aligned}
I I I \leq & C\left(\varepsilon\left|u_{h}\right|_{H^{1}(T)}^{2}+\varepsilon h^{-1}\left|u_{h}\right|_{H^{1}(T)}\left\|u_{h}\right\|_{L^{2}(T)}\right) \\
& +C\left(\varepsilon^{1 / 2}\left|u_{h}\right|_{H^{1}(T)}\right)\left(\varepsilon^{1 / 2}\left|z_{h}\right|_{H^{1}(T)}+\varepsilon^{-1 / 2}\left\|z_{h}\right\|_{L^{2}(T)}\right) \\
& +C\left(h^{1 / 2}\|f\|_{L^{2}(T)}\right)\left(h^{-1 / 2}\left\|u_{h}\right\|_{L^{2}(T)}+\varepsilon^{-1 / 2}\left\|z_{h}\right\|_{L^{2}(T)}\right) ;
\end{aligned}
$$

then, using in (2.31) the equivalences (2.30) and (2.33) we infer

$$
\begin{aligned}
\left\|u_{h}\right\|_{L^{2}(\partial T)}^{2} \leq & C\left(\varepsilon\left|u_{h}\right|_{H^{1}(T)}^{2}+h^{-1}\left\|u_{h}\right\|_{L^{2}(T)}^{2}+h\|f\|_{L^{2}(T)}^{2}\right) \\
& +C\left(\varepsilon^{1 / 2}\left|u_{h}\right|_{H^{1}(T)}+h^{1 / 2}\|f\|_{L^{2}(T)}\right)\left(\left\|u_{h}\right\|_{L^{2}(\partial T)}\right),
\end{aligned}
$$

which concludes the proof of (2.26).

In order to prove (2.27) we proceed similarly: considering $e$ instead of $u_{h}$ in (2.31) and (2.32) we have

$$
\int_{\partial T} \mathbf{c} \cdot \mathbf{n} e^{2} \omega d \sigma(\mathbf{x}) \leq C h^{-1}\|e\|_{L^{2}(T)}^{2}+\int_{T} \mathbf{c} \cdot \nabla\left(e^{2}\right) \omega d \mathbf{x} .
$$

In this case, still using Corollary 2.3, we get a new $z_{h}$ such that $z_{h}=e$ on $\partial T$ and

$$
\varepsilon^{1 / 2}\left|z_{h}\right|_{H^{1}(T)}+\varepsilon^{-1 / 2}\left\|z_{h}\right\|_{L^{2}(T)} \leq C\|e\|_{L^{2}(\partial T)} .
$$

Now, by virtue of (2.20), we have

$$
\begin{aligned}
\varepsilon \int_{T} \nabla e \cdot \nabla\left(\omega e-\omega z_{h}\right) d \mathbf{x}+\int_{T} \mathbf{c} \cdot \nabla e\left(\omega e-\omega z_{h}\right) d \mathbf{x} \\
\quad=-\varepsilon \int_{T} \nabla \eta \cdot \nabla\left(\omega e-\omega z_{h}\right) d \mathbf{x}-\int_{T} \mathbf{c} \cdot \nabla \eta\left(\omega e-\omega z_{h}\right) d \mathbf{x}
\end{aligned}
$$

and so, after integration by parts

$$
\begin{aligned}
\frac{1}{2} \int_{T} \mathbf{c} \cdot \nabla\left(e^{2}\right) \omega d \mathbf{x}= & \int_{T} e \mathbf{c} \cdot \nabla e \omega d \mathbf{x} \\
= & \varepsilon \int_{T} \nabla e \cdot \nabla\left(\omega z_{h}-\omega e\right) d \mathbf{x}+\int_{T} z_{h} \mathbf{c} \cdot \nabla e \omega d \mathbf{x} \\
& +\varepsilon \int_{T} \nabla \eta \cdot \nabla\left(\omega z_{h}-\omega e\right) d \mathbf{x}-\int_{T} \eta \mathbf{c} \cdot \nabla\left(\omega z_{h}-\omega e\right) d \mathbf{x} \\
= & I V+V+V I+V I I .
\end{aligned}
$$

For those terms in this last sum which do not include $\eta$ we have, as in the previous case,

$$
\begin{aligned}
I V+V \leq & C\left(\varepsilon|e|_{H^{1}(T)}^{2}+\varepsilon h^{-1}|e|_{H^{1}(T)}\|e\|_{L^{2}(T)}\right) \\
& +C\left(\varepsilon^{1 / 2}|e|_{H^{1}(T)}\right)\left(\|e\|_{L^{2}(\partial T)}\right),
\end{aligned}
$$


while, using for $\eta$ the error inequality (2.16)

$$
\begin{aligned}
V I+V I I \leq & C\left(\varepsilon^{1 / 2}|e|_{H^{1}(T)}+h^{-1 / 2}\|e\|_{L^{2}(T)}\right)\left(h^{r-1 / 2}|u|_{H^{r}(\mathcal{N}(T))}\right) \\
& +C\left(\|e\|_{L^{2}(\partial T)}\right)\left(h^{r-1 / 2}|u|_{H^{r}(\mathcal{N}(T))}\right) .
\end{aligned}
$$

Hence, using the equivalence (2.30) in (2.35), we get (2.27).

3. Stability and global error analysis. This section is devoted to global stability, convergence and error estimates for the RFB method. The stability analysis, besides being a crucial step in the derivation of the error bounds, is in any case of interest since it allows to obtain a convergence result without any extra regularity hypotheses on the exact solution $u$. The error estimate is a generalization of Theorem 2.5. The structure of these proofs reflects that of Theorem 2.5 with some technical complication that is needed in order to obtain the extra $L^{2}$-norm control.

The assumptions (1.2) and (2.2) guarantee the existence of a smooth gradient field $\delta: \Omega \rightarrow \mathbb{R}$ such that $\mathbf{c} \cdot \nabla \delta \geq C>0$, with $C=C(\Omega, \mathbf{c}$ ) (see, for example, Lemma 3.2 in [15]). Then, as usual, we define

$$
\phi:=\exp (-\delta) \quad \text { in } \Omega,
$$

for which there exist constants $\alpha_{i}=\alpha_{i}(\Omega, \mathbf{c})$ such that

$$
\begin{array}{clrl}
\alpha_{1} \leq \phi & \leq \alpha_{2} & & \text { in } \Omega, \\
|\nabla \phi| & \leq \alpha_{3} \phi & & \text { in } \Omega, \\
-\mathbf{c} \cdot \nabla \phi & \geq 2 \alpha_{4} \phi & & \text { in } \Omega .
\end{array}
$$

These properties allow us to improve the coercivity condition (2.22) in the following well known lemma.

Lemma 3.1. Suppose that (1.2) holds true and let $\phi$ be defined by (3.1). Then there exist constants $C_{1}=C_{1}(\Omega, \mathbf{c})$ and $C_{2}=C_{2}(\Omega, \mathbf{c})$ such that for each $\varepsilon \leq C_{1}$ we have:

$$
a(v, \phi v) \geq C_{2}\left(\varepsilon|v|_{H^{1}(\Omega)}^{2}+\|v\|_{L^{2}(\Omega)}^{2}\right) \quad \forall v \in H_{0}^{1}(\Omega) .
$$

We have to pay a price for the introduction of the weight function $\phi$. Indeed the product of $\phi$ times a function of $V_{h}$ does not belong to $V_{h}$, and we cannot use it as a test function. In order to overcome this difficulty we state a specific approximation result.

Lemma 3.2. Assume that (1.2), (2.1), (2.2), (2.3) and (2.4) (or (2.5) in the case $k=1$ ) hold true; let $u_{h}$ be the solution of (1.10) and $\phi$ be defined by (3.1). Then there exists a constant $C=C(\Omega, \mathcal{T}, \mathbf{c}, k)$ such that

$$
\begin{gathered}
\varepsilon\left|\phi u_{h}-\Pi_{h, \varepsilon}\left(\phi u_{h}\right)\right|_{H^{1}(\Omega)}^{2}+\varepsilon^{-1}\left\|\phi u_{h}-\Pi_{h, \varepsilon}\left(\phi u_{h}\right)\right\|_{L^{2}(\Omega)}^{2} \\
\leq C h\left(\left\|u_{h}\right\|_{L^{2}(\Omega)}^{2}+\varepsilon h\left|u_{h}\right|_{H^{1}(\Omega)}^{2}+h^{2}\|f\|_{L^{2}(\Omega)}^{2}\right) .
\end{gathered}
$$

Moreover let $u$ be the solution of (1.4), let e be defined as in (2.18) and suppose that $u \in H^{r}(\Omega)$, with $1 \leq r \leq k+1$. Then

$$
\begin{aligned}
\varepsilon \mid \phi e & -\left.\Pi_{h, \varepsilon}(\phi e)\right|_{H^{1}(\Omega)} ^{2}+\varepsilon^{-1}\left\|\phi e-\Pi_{h, \varepsilon}(\phi e)\right\|_{L^{2}(\Omega)}^{2} \\
& \leq C h\left(\|e\|_{L^{2}(\Omega)}^{2}+\varepsilon h|e|_{H^{1}(\Omega)}^{2}+h^{2 r}|u|_{H^{r}(\Omega)}^{2}\right) .
\end{aligned}
$$


Proof. In the following proof of inequalities (3.6) and (3.7), $C$ and $C_{i}$ denote constants that depend only on $\Omega, \mathcal{T}$, $\mathbf{c}$ and $k$.

Let us prove first the inequality (3.6). Let $T$ be a generic triangle in $\mathcal{T}_{h}$. Since $\phi$ is a fixed and regular function, and since $u_{h}$ is polynomial of degree $k$ on $\partial T_{i}$, we deduce a so called super-approximation estimate: denoting by $\mathbf{v}_{i}$ the direction of $\partial T_{i}$ we have

$$
\begin{aligned}
\left|\phi u_{h}\right|_{H^{k+1}\left(\partial T_{i}\right)}^{2} & =\int_{\partial T_{i}}\left(D_{\mathbf{v}_{i}}^{k+1}\left(\phi u_{h}\right)\right)^{2} d \sigma(\mathbf{x}) \\
& \leq C \int_{\partial T_{i}} \sum_{\substack{m, n \geq 0 \\
m+n=k+1}}\left(D_{\mathbf{v}_{i}}^{m} \phi D_{\mathbf{v}_{i}}^{n} u_{h}\right)^{2} d \sigma(\mathbf{x}) \\
& \leq C \sum_{0 \leq n \leq k}\left|u_{h}\right|_{H^{n}\left(\partial T_{i}\right)}^{2} .
\end{aligned}
$$

Using then in (3.8) the inverse inequality (2.10) and substituting in (2.15) we obtain

$$
\varepsilon\left|\phi u_{h}-\Pi_{h, \varepsilon}\left(\phi u_{h}\right)\right|_{H^{1}(T)}^{2}+\varepsilon^{-1}\left\|\phi u_{h}-\Pi_{h, \varepsilon}\left(\phi u_{h}\right)\right\|_{L^{2}(T)}^{2} \leq C h^{2}\left\|u_{h}\right\|_{L^{2}(\partial T)}^{2} ;
$$

the estimate (3.6) simply follows from (2.26) and addition over all triangles.

In the same way we obtain a constant $C$ such that

$$
\varepsilon\left|\phi e-\Pi_{h, \varepsilon}(\phi e)\right|_{H^{1}(T)}^{2}+\varepsilon^{-1}\left\|\phi e-\Pi_{h, \varepsilon}(\phi e)\right\|_{L^{2}(T)}^{2} \leq C h^{2}\|e\|_{L^{2}(\partial T)}^{2},
$$

so that using (2.27) and summing over all triangles we obtain (3.7).

Now we can state our stability result.

Theorem 3.3. Assume that (1.2), (2.1), (2.2), (2.3) and (2.4) (or (2.5) in the case $k=1$ ) are fulfilled and let $u_{h}$ be the solution of (1.10). Then there exist constants $C_{1}=C_{1}(\Omega, \mathcal{T}, \mathbf{c}, k)$ and $C_{2}=C_{2}(\Omega, \mathcal{T}, \mathbf{c}, k)$ such that for each $h \leq C_{1}$, the $R F B$ method verifies the stability inequality

$$
\varepsilon^{1 / 2}\left|u_{h}\right|_{H^{1}(\Omega)}+\left\|u_{h}\right\|_{L^{2}(\Omega)} \leq C_{2}\|f\|_{L^{2}(\Omega)} .
$$

Proof. In the following proof we shall assume that the constants $C$ and $C_{i}$ are dependent only on $\Omega, \mathcal{T}$, $\mathbf{c}$ and $k$. Supposing that $\varepsilon$ is small enough (as in the hypotheses of Lemma 3.1) from (3.5) we have

$$
C\left(\varepsilon\left|u_{h}\right|_{H^{1}(\Omega)}^{2}+\left\|u_{h}\right\|_{L^{2}(\Omega)}^{2}\right) \leq a\left(u_{h}, \phi u_{h}\right),
$$

where $\phi$ is defined by (3.1). We split the right hand side of (3.11) as follows:

$$
\begin{aligned}
a\left(u_{h}, \phi u_{h}\right) & =a\left(u_{h}, \Pi_{h, \varepsilon}\left(\phi u_{h}\right)\right)+a\left(u_{h}, \phi u_{h}-\Pi_{h, \varepsilon}\left(\phi u_{h}\right)\right) \\
& =I+I I .
\end{aligned}
$$

From (1.10) we obtain

$$
I=\left\langle f, \Pi_{h, \varepsilon}\left(\phi u_{h}\right)\right\rangle
$$


using the triangle inequality, (3.2) and (3.6) we have

$$
\begin{aligned}
\left\|\Pi_{h, \varepsilon}\left(\phi u_{h}\right)\right\|_{L^{2}(\Omega)} & \leq\left\|\phi u_{h}-\Pi_{h, \varepsilon}\left(\phi u_{h}\right)\right\|_{L^{2}(\Omega)}+\left\|\phi u_{h}\right\|_{L^{2}(\Omega)} \\
& \leq C\left(\left\|u_{h}\right\|_{L^{2}(\Omega)}+\varepsilon^{1 / 2}\left|u_{h}\right|_{H^{1}(\Omega)}+\|f\|_{L^{2}(\Omega)}\right) .
\end{aligned}
$$

Then

$$
I \leq C\|f\|_{L^{2}(\Omega)}\left(\left\|u_{h}\right\|_{L^{2}(\Omega)}+\varepsilon^{1 / 2}\left|u_{h}\right|_{H^{1}(\Omega)}\right)+C\|f\|_{L^{2}(\Omega)}^{2} .
$$

Using again the approximation inequality (3.6) we have

$$
\begin{aligned}
I I \leq & C\left(\varepsilon^{1 / 2}\left|u_{h}\right|_{H^{1}(\Omega)}\right)\left(\varepsilon^{-1 / 2}\left\|\phi u_{h}-\Pi_{h, \varepsilon}\left(\phi u_{h}\right)\right\|_{L^{2}(\Omega)}\right) \\
& +C\left(\varepsilon^{1 / 2}\left|u_{h}\right|_{H^{1}(\Omega)}\right)\left(\varepsilon^{1 / 2}\left|\phi u_{h}-\Pi_{h, \varepsilon}\left(\phi u_{h}\right)\right|_{H^{1}(\Omega)}\right) \\
\leq & C h^{1 / 2}\left(\varepsilon^{1 / 2}\left|u_{h}\right|_{H^{1}(\Omega)}\right) \\
& \cdot\left(\left\|u_{h}\right\|_{L^{2}(\Omega)}+\sqrt{\varepsilon h}\left|u_{h}\right|_{H^{1}(\Omega)}+h\|f\|_{L^{2}(\Omega)}\right) \\
\leq & C h^{1 / 2}\left(\varepsilon\left|u_{h}\right|_{H^{1}(\Omega)}^{2}+\left\|u_{h}\right\|_{L^{2}(\Omega)}^{2}\right)+C \varepsilon^{1 / 2}\left|u_{h}\right|_{H^{1}(\Omega)}\|f\|_{L^{2}(\Omega)},
\end{aligned}
$$

where in the last addendum we have removed the factor $h^{3 / 2}$, since it is bounded thanks to (2.3). By virtue of (3.11)-(3.13) we get

$$
\begin{aligned}
& \left(C_{1}-h^{1 / 2}\right)\left(\varepsilon\left|u_{h}\right|_{H^{1}(\Omega)}^{2}+\left\|u_{h}\right\|_{L^{2}(\Omega)}^{2}\right) \\
& \quad \leq C_{2}\|f\|_{L^{2}(\Omega)}^{2}+C_{2}\left(\left\|u_{h}\right\|_{L^{2}(\Omega)}+\varepsilon^{1 / 2}\left|u_{h}\right|_{H^{1}(\Omega)}\right)\|f\|_{L^{2}(\Omega)},
\end{aligned}
$$

then, choosing $h$ small enough, we get (3.10).

The stability property just proved allows us to obtain the following convergence result, uniform in $\varepsilon$, that does not require any assumption on the regularity of the exact solution $u$.

Theorem 3.4. Assume that (1.2), (2.1), (2.2), (2.3) and (2.4) (or (2.5) in the case $k=1$ ) are fulfilled and let $u$ and $u_{h}$ be the solutions of (1.4) and (1.10) respectively. Then there exist constants $C_{1}=C_{1}(\Omega, \mathcal{T}, \mathbf{c}, k)$ and $C_{2}=C_{2}(\Omega, \mathcal{T}, \mathbf{c}, k)$ such that for each $h \leq C_{1}$ the RFB method verifies

$$
\left\|\mathbf{c} \cdot \nabla\left(u-u_{h}\right)\right\|_{H^{-1}(\Omega)} \leq C_{2} h^{1 / 2}\|f\|_{L^{2}(\Omega)} .
$$

Proof. It follows directly from (2.24), the stability conditions (1.5) and (3.10).

Now we can complete the global error analysis in the $L^{2}$-norm.

Theorem 3.5. Assume that (1.2), (2.1), (2.2), (2.3) and (2.4) (or (2.5) in the case $k=1$ ) are fulfilled, let $u$ and $u_{h}$ be the solutions of (1.4) and (1.10) respectively and suppose that $u \in H^{r}(\Omega)$ with $1 \leq r \leq k+1$. Then there exist constants $C_{1}=$ $C_{1}(\Omega, \mathcal{T}, \mathbf{c}, k)$ and $C_{2}=C_{2}(\Omega, \mathcal{T}, \mathbf{c}, k)$ such that for each $h \leq C_{1}$

$$
\left\|u-u_{h}\right\|_{L^{2}(\Omega)} \leq C_{2} h^{r-1 / 2}|u|_{H^{r}(\Omega)} .
$$


Proof. In the following proof we shall assume that the constants $C$ and $C_{i}$ are dependent only on $\Omega, \mathcal{T}, \mathbf{c}$ and $k$. Let $e$ and $\eta$ be defined by (2.18) and (2.19). We have

$$
\left\|u-u_{h}\right\|_{L^{2}(\Omega)} \leq\|e\|_{L^{2}(\Omega)}+\|\eta\|_{L^{2}(\Omega)},
$$

and estimate (2.23) yields

$$
\|\eta\|_{L^{2}(\Omega)} \leq \varepsilon^{1 / 2} h^{r-1 / 2}|u|_{H^{r}(\Omega)},
$$

so it remains only to estimate $\|e\|_{L^{2}(\Omega)}$. This can be done with the same technique as in Theorem 3.3 (we include the details for the reader's convenience): taking $\varepsilon$ small enough, as in the hypothesis of Lemma 3.1, and using the Galerkin property (2.20) we have

$$
\begin{aligned}
C\left(\varepsilon|e|_{H^{1}(\Omega)}^{2}+\|e\|_{L^{2}(\Omega)}^{2}\right) \leq & a(e, \phi e) \\
= & a\left(e, \Pi_{h, \varepsilon}(\phi e)\right)+a\left(e, \phi e-\Pi_{h, \varepsilon}(\phi e)\right) \\
= & -a\left(\eta, \Pi_{h, \varepsilon}(\phi e)\right)+a\left(e, \phi e-\Pi_{h, \varepsilon}(\phi e)\right) \\
= & -a(\eta, \phi e)+a\left(\eta, \phi e-\Pi_{h, \varepsilon}(\phi e)\right) \\
& +a\left(e, \phi e-\Pi_{h, \varepsilon}(\phi e)\right) \\
= & I+I I+I I I .
\end{aligned}
$$

Using integration by parts and (2.23) on the first term we have

$$
\begin{aligned}
I= & -\varepsilon \int_{\Omega} \nabla \eta \cdot \nabla(\phi e) d \mathbf{x}+\int_{\Omega} \eta \mathbf{c} \cdot \nabla(\phi e) d \mathbf{x} \\
= & -\varepsilon \int_{\Omega} e \nabla \eta \cdot \nabla \phi d \mathbf{x}-\varepsilon \int_{\Omega} \phi \nabla \eta \cdot \nabla e d \mathbf{x} \\
& +\int_{\Omega} e \eta \mathbf{c} \cdot \nabla \phi d \mathbf{x}+\int_{\Omega} \phi \eta \mathbf{c} \cdot \nabla e d \mathbf{x} \\
\leq & C\left(\varepsilon^{1 / 2}|e|_{H^{1}(\Omega)}+\|e\|_{L^{2}(\Omega)}\right) \\
& \cdot\left(\varepsilon^{1 / 2}|\eta|_{H^{1}(\Omega)}+\varepsilon^{-1 / 2}\|\eta\|_{L^{2}(\Omega)}\right) \\
\leq & C\left(\varepsilon^{1 / 2}|e|_{H^{1}(\Omega)}+\|e\|_{L^{2}(\Omega)}\right)\left(h^{r-1 / 2}|u|_{H^{r}(\Omega)}\right) .
\end{aligned}
$$

Moreover, using (3.7) and (2.23), we get

$$
\begin{aligned}
I I= & \varepsilon \int_{\Omega} \nabla \eta \cdot \nabla\left(\phi e-\Pi_{h, \varepsilon}(\phi e)\right) d \mathbf{x}+\int_{\Omega} \mathbf{c} \cdot \nabla \eta\left(\phi e-\Pi_{h, \varepsilon}(\phi e)\right) d \mathbf{x} \\
\leq & C h^{1 / 2}\left(\|e\|_{L^{2}(\Omega)}+\varepsilon^{1 / 2} h^{1 / 2}|e|_{H^{1}(\Omega)}+h^{r}|u|_{H^{r}(\Omega)}\right) \\
& \cdot\left(\varepsilon^{1 / 2}|\eta|_{H^{1}(\Omega)}\right) \\
\leq & C\left(\|e\|_{L^{2}(\Omega)}+\varepsilon^{1 / 2}|e|_{H^{1}(\Omega)}\right)\left(h^{r-1 / 2}|u|_{H^{r}(\Omega)}\right) \\
& +C h^{2 r-1}|u|_{H^{r}(\Omega)}^{2},
\end{aligned}
$$


and

$$
\begin{aligned}
I I I= & \varepsilon \int_{\Omega} \nabla e \cdot \nabla\left(\phi e-\Pi_{h, \varepsilon}(\phi e)\right) d \mathbf{x}+\int_{\Omega} \mathbf{c} \cdot \nabla e\left(\phi e-\Pi_{h, \varepsilon}(\phi e)\right) d \mathbf{x} \\
\leq & C h^{1 / 2}\left(\|e\|_{L^{2}(\Omega)}+\varepsilon^{1 / 2} h^{1 / 2}|e|_{H^{1}(\Omega)}+h^{r-1 / 2}|u|_{H^{r}(\Omega)}\right) \\
& \cdot\left(\varepsilon^{1 / 2}|e|_{H^{1}(\Omega)}\right) \\
\leq & C h^{1 / 2}\left(\|e\|_{L^{2}(\Omega)}+\varepsilon^{1 / 2}|e|_{H^{1}(\Omega)}\right)\left(\varepsilon^{1 / 2}|e|_{H^{1}(\Omega)}\right) \\
& +C\left(h^{r-1 / 2}|u|_{H^{r}(\Omega)}\right)\left(\varepsilon^{1 / 2}|e|_{H^{1}(\Omega)}\right) .
\end{aligned}
$$

In conclusion we obtain

$$
\begin{aligned}
\left(C_{1}-\right. & \left.h^{1 / 2}\right)\left(\varepsilon|e|_{H^{1}(\Omega)}^{2}+\|e\|_{L^{2}(\Omega)}^{2}\right) \\
\leq & C_{2}\left(\|e\|_{L^{2}(\Omega)}+\varepsilon^{1 / 2}|e|_{H^{1}(\Omega)}\right)\left(h^{r-1 / 2}|u|_{H^{r}(\Omega)}\right) \\
& +C_{2} h^{2 r-1}|u|_{H^{r}(\Omega)}^{2},
\end{aligned}
$$

so, choosing $h$ small enough, we get

$$
\|e\|_{L^{2}(\Omega)}+\varepsilon^{1 / 2}|e|_{H^{1}(\Omega)} \leq C h^{r-1 / 2}|u|_{H^{r}(\Omega)},
$$

and this concludes the proof.

REMARK 3.6. The order of convergence of the error with respect to the $L^{2}$-norm is the same for the RFB and SUPG methods, both being one half order suboptimal. In our opinion this bound cannot be improved on general meshes. This is confirmed for the SUPG method by numerical experiments (see [22]). We postpone to a further work numerical investigations to assess the optimality of the estimate for RFB.

4. Local error analysis. Following the same structure of the previous section, we now take a proper cut-off function $\psi$ (depending on $h$ ) instead of the weight function $\phi$, in order to obtain a localized error estimate. This technique is often used in the local analysis of both exact and numerical solutions to advection-dominated problems (see [18], [23] and in particular [19]).

In the following $\overline{\mathbf{c}}$ is a field such that $|\overline{\mathbf{c}}|=1$ in $\Omega$ and $\overline{\mathbf{c}} \cdot \mathbf{c}=0$ in $\Omega$.

LEMMA 4.1. Let $s$ and $\gamma$ be real constants with $s, \gamma \geq 1$, let $\Omega^{\prime \prime}$ be a subdomain of $\Omega$ with piecewise smooth boundary $\partial \Omega^{\prime \prime}=\left(\partial \Omega^{\prime \prime}\right)^{-} \cup\left(\partial \Omega^{\prime \prime}\right)^{0} \cup\left(\partial \Omega^{\prime \prime}\right)^{+}$such that, denoting by $\mathbf{n}$ the outward normal vector,

$$
\begin{aligned}
\left(\partial \Omega^{\prime \prime}\right)^{-} & :=\left\{\mathbf{x} \in \partial \Omega^{\prime \prime}: \mathbf{c} \cdot \mathbf{n}<0\right\} \\
\left(\partial \Omega^{\prime \prime}\right)^{0} & :=\left\{\mathbf{x} \in \partial \Omega^{\prime \prime}: \mathbf{c} \cdot \mathbf{n}=0\right\} \\
\left(\partial \Omega^{\prime \prime}\right)^{+} & :=\left\{\mathbf{x} \in \partial \Omega^{\prime \prime}: \mathbf{c} \cdot \mathbf{n}>0\right\}
\end{aligned}
$$

Suppose that $\left(\partial \Omega^{\prime \prime}\right)^{-} \subset \partial \Omega$, that all points upstream of a point on $\left(\partial \Omega^{\prime \prime}\right)^{0}$ belong to $\left(\partial \Omega^{\prime \prime}\right)^{0}$ and that $|\mathbf{c} \cdot \mathbf{n}| \geq C>0$ on $\left(\partial \Omega^{\prime \prime}\right)^{-} \cup\left(\partial \Omega^{\prime \prime}\right)^{+}$. Assume that (1.2), (2.2) hold true. Then there exist constants $\beta_{1}=\beta_{1}\left(\Omega^{\prime \prime}\right), \beta_{2}=\beta_{2}\left(\mathbf{c}, \Omega^{\prime \prime}\right)$ and $\beta_{3}=\beta_{3}\left(s, k, \mathbf{c}, \Omega^{\prime \prime}\right)$ such that, if $\Omega^{\prime}$ is a sub-domain of $\Omega^{\prime \prime}$ at distance at least 
$\beta_{3} \gamma h \ln h^{-1}$ and $\beta_{3} \gamma h^{1 / 2} \ln h^{-1}$ from $\left(\partial \Omega^{\prime \prime}\right)^{+}$and $\left(\partial \Omega^{\prime \prime}\right)^{0}$ respectively, then there exists a function $\psi$ with the following properties:

$$
\begin{aligned}
\psi & \leq \beta_{1} & & \text { in } \Omega, \\
\psi & \geq 0 & & \text { in } \Omega, \\
\psi & \geq 1 & & \text { in } \Omega^{\prime}, \\
\psi & =0 & & \text { in } \Omega \backslash \Omega^{\prime \prime}, \\
-D_{\mathbf{c}} \psi & \geq \psi & & \text { in } \Omega, \\
\max _{|\mathbf{y}| \leq \gamma h} \frac{\psi(\mathbf{x}+\mathbf{y})}{\psi(\mathbf{x})} & \leq \beta_{2} & & \text { in }\left\{\mathbf{x} \in \Omega: \psi(\mathbf{x}) \geq \beta_{2} h^{2 s}\right\}, \\
\max _{|\mathbf{y}| \leq \gamma h} \frac{-D_{\mathbf{c}} \psi(\mathbf{x}+\mathbf{y})}{-D_{\mathbf{c}} \psi(\mathbf{x})} & \leq \beta_{2} & & \text { in }\left\{\mathbf{x} \in \Omega:-D_{\mathbf{c}} \psi(\mathbf{x}) \geq \beta_{2} h^{2 s}\right\} ;
\end{aligned}
$$

for each positive integer $i \leq k+1$

$$
\begin{array}{ll}
\left|D_{\mathbf{c}}^{i} \psi\right| \leq \beta_{2}\left(\gamma^{-1} h^{-i} \psi+h^{2 s}\right) & \text { in } \Omega, \\
\left|D_{\mathbf{c}}^{i} \psi\right| \leq \beta_{2}\left(\gamma^{-1} h^{-i / 2} \psi-h^{1-i} D_{\mathbf{c}} \psi+h^{2 s}\right) & \text { in } \Omega ;
\end{array}
$$

for each integers $i, j: 1 \leq i+j \leq k$

$$
\left|D_{\mathbf{c}}^{i+1} D_{\overline{\mathbf{c}}}^{j} \psi\right| \leq \beta_{2}\left(-\gamma^{-1} h^{-(i+j)} D_{\mathbf{c}} \psi+h^{2 s}\right) \quad \text { in } \Omega .
$$

Proof. We refer to Lemma 2.1 in [19] for a detailed construction.

We shall use in the following weighted norms based on the weight $\psi$ given by the previous Lemma 4.1. Note that $\psi$ depends on the constants $s$ and $\gamma$ : the role of $s$ will be discussed before Theorem 4.4, while $\gamma$ will be chosen in the sequel. These constants are independent of $h$ and $\varepsilon$. We only assume the condition:

$$
\varepsilon \leq h \leq \gamma^{-1} \leq 1
$$

The weight function will be indicated as in the following examples:

$$
\begin{aligned}
\|v\|_{L^{2}(T, \psi)}^{2} & :=\int_{T} v^{2} \psi d \mathbf{x}, \\
|v|_{H^{1}(T, \psi)}^{2} & :=\int_{T}|\nabla v|^{2} \psi d \mathbf{x},
\end{aligned}
$$

and similarly in the case of

$$
\|v\|_{L^{2}(\partial T, \psi)}^{2}:=\int_{\partial T} v^{2} \psi d \sigma(\mathbf{x}) .
$$

Moreover, given an open set $\Psi$ contained in $\Omega$ we denote by $\mathcal{C}(\Psi)$ (respectively by $\mathcal{N}(\Psi)$ ) the union of $\bar{T}$ (respectively the union of $\mathcal{N}(T)$ ) for all the triangles $T$ in $\mathcal{T}_{h}$ intersecting $\Psi$.

We can now state the following coercivity condition. 
Lemma 4.2. Assume the hypotheses and notation of Lemma 4.1 and the conditions (1.2), (4.11). There exist constants $C_{1}=C_{1}(\Omega, \mathbf{c})$ and $C_{2}=C_{2}(\Omega, \mathbf{c})$ such that if $\gamma \geq C_{1}$ then

$$
\begin{aligned}
& a(v, \psi v)+h^{2 s}\left(\varepsilon|v|_{H^{1}\left(\Omega^{\prime \prime}\right)}^{2}+\|v\|_{L^{2}\left(\Omega^{\prime \prime}\right)}^{2}\right) \\
& \quad \geq C_{2}\left(\varepsilon|v|_{H^{1}(\Omega, \psi)}^{2}+\|v\|_{L^{2}\left(\Omega,-D_{\mathbf{c}} \psi\right)}^{2}\right) \quad \forall v \in H_{0}^{1}(\Omega) .
\end{aligned}
$$

Proof. The constant $C$ in the proof depends only on $\Omega$ and c. Using (4.4) we split

$$
\begin{aligned}
a(v, \psi v) & =\varepsilon \int_{\Omega^{\prime \prime}}|\nabla v|^{2} \psi d \mathbf{x}+\varepsilon \int_{\Omega^{\prime \prime}} v \nabla v \cdot \nabla \psi d \mathbf{x}+\int_{\Omega^{\prime \prime}} \psi \mathbf{c} \cdot \nabla\left(\frac{v^{2}}{2}\right) d \mathbf{x} \\
& =I+I I+I I I .
\end{aligned}
$$

Obviously

$$
I=\varepsilon|v|_{H^{1}(T, \psi)}^{2} ;
$$

moreover integration by parts and (1.2) yield

$$
\begin{aligned}
I I I & =-\int_{\Omega^{\prime \prime}} \operatorname{div}(\mathbf{c}) \frac{v^{2}}{2} \psi d \mathbf{x}-\int_{\Omega^{\prime \prime}} \mathbf{c} \cdot \nabla \psi \frac{v^{2}}{2} d \mathbf{x} \\
& \geq-\frac{1}{2} \int_{\Omega^{\prime \prime}} v^{2} \mathbf{c} \cdot \nabla \psi d \mathbf{x} \\
& =\frac{1}{2}\|v\|_{L^{2}\left(\Omega,-D_{\mathbf{c}} \psi\right)}^{2} .
\end{aligned}
$$

From (4.9) we have

$$
\begin{aligned}
|\nabla \psi| & \leq C\left(\left|D_{\mathbf{c}} \psi\right|+\mid D_{\overline{\mathbf{c}} \psi} \psi\right) \\
& \leq C\left(-D_{\mathbf{c}} \psi+\gamma^{-1} h^{-1 / 2} \psi+h^{2 s}\right),
\end{aligned}
$$

so that the second term verifies

$$
\begin{aligned}
|I I| \leq & C \int_{\Omega^{\prime \prime}} \varepsilon^{1 / 2}|\nabla v||v|\left(\varepsilon^{1 / 2}\left|D_{\mathbf{c}} \psi\right|+\varepsilon^{1 / 2} h^{-1 / 2} \gamma^{-1} \psi+\varepsilon^{1 / 2} h^{2 s}\right) d \mathbf{x} \\
\leq & C\left(\varepsilon^{1 / 2}|v|_{H^{1}\left(\Omega,-\varepsilon D_{\mathbf{c}} \psi\right)}\right)\left(\|v\|_{L^{2}\left(\Omega,-D_{\mathbf{c}} \psi\right)}\right) \\
& +C \gamma^{-1}\left(\varepsilon^{1 / 2}|v|_{H^{1}(\Omega, \psi)}\right)\left(\|v\|_{L^{2}(\Omega, \psi)}\right) \\
& +C \varepsilon^{1 / 2} h^{2 s}\left(\varepsilon^{1 / 2}|v|_{H^{1}\left(\Omega^{\prime \prime}\right)}\right)\left(\|v\|_{L^{2}\left(\Omega^{\prime \prime}\right)}\right) \\
= & I V+V+V I .
\end{aligned}
$$

From (4.8) we have

$$
\begin{aligned}
I V \leq & C \gamma^{-1 / 2}\left(\varepsilon^{1 / 2}|v|_{H^{1}(\Omega, \psi)}\right)\left(\|v\|_{L^{2}\left(\Omega,-D_{\mathbf{c}} \psi\right)}\right) \\
& +C \varepsilon^{1 / 2} h^{s}\left(\varepsilon^{1 / 2}|v|_{H^{1}\left(\Omega^{\prime \prime}\right)}\right)\left(\|v\|_{L^{2}\left(\Omega,-D_{\mathbf{c}} \psi\right)}\right) \\
\leq & C \gamma^{-1 / 2}\left(\varepsilon|v|_{H^{1}(\Omega, \psi)}^{2}+\|v\|_{L^{2}\left(\Omega,-D_{\mathbf{c}} \psi\right)}^{2}\right)+C \varepsilon \gamma^{1 / 2} h^{2 s}|v|_{H^{1}\left(\Omega^{\prime \prime}\right)}^{2}
\end{aligned}
$$


and from (4.5) we have

$$
V \leq C \gamma^{-1}\left(\varepsilon^{1 / 2}|v|_{H^{1}(\Omega, \psi)}\right)\left(\|v\|_{L^{2}\left(\Omega,-D_{\mathbf{c}} \psi\right)}\right) .
$$

Finally we get

$$
\begin{aligned}
|I I| \leq & C \gamma^{-1 / 2}\left(\varepsilon|v|_{H^{1}(\Omega, \psi)}^{2}+\|v\|_{L^{2}\left(\Omega,-D_{\mathbf{c}} \psi\right)}^{2}\right) \\
& +C h^{2 s}\left(\varepsilon^{1 / 2}+\varepsilon \gamma^{1 / 2}\right)\left(\varepsilon|v|_{H^{1}\left(\Omega^{\prime \prime}\right)}^{2}+\|v\|_{L^{2}\left(\Omega^{\prime \prime}\right)}^{2}\right) \\
\leq & C \gamma^{-1 / 2}(I+I I I)+C h^{2 s}\left(\varepsilon^{1 / 2}+\varepsilon \gamma^{1 / 2}\right)\left(\varepsilon|v|_{H^{1}\left(\Omega^{\prime \prime}\right)}^{2}+\|v\|_{L^{2}\left(\Omega^{\prime \prime}\right)}^{2}\right) .
\end{aligned}
$$

By (4.11) we have $\varepsilon^{1 / 2}+\varepsilon \gamma^{1 / 2} \leq 2 \gamma^{-1 / 2} \leq 2$; hence (4.12) is verified when $\gamma$ is large enough.

Now we state the analogue of Lemma 3.2. In this case there are more technical complications, because the cut-off function $\psi$ depends on $h$.

Lemma 4.3. Assume that (1.2), (2.1), (2.2), (2.3), (2.4) or (2.5) in the case $k=1$, (4.11) are fulfilled, with the hypotheses and notation given in Lemma 4.1. Let $u$ be the solution of (1.4) and let e be defined as in (2.18). Define

$$
\mathcal{T}_{h}^{\prime}:=\left\{T \in \mathcal{T}_{h}: \psi(\mathbf{x}) \geq \beta_{2} h^{2 s}, \forall \mathbf{x} \in T\right\} .
$$

and

$$
\mathcal{T}_{h}^{\prime \prime}:=\mathcal{T}_{h} \backslash \mathcal{T}_{h}^{\prime} .
$$

Then there exist positive constants $C_{1}=C_{1}(\mathcal{T})$ and $C_{2}=C_{2}\left(\Omega^{\prime \prime}, \mathcal{T}, \mathbf{c}, k, s\right)$ such that for $\gamma \geq C_{1}$ :

$$
\begin{aligned}
\sum_{T \in \mathcal{T}_{h}^{\prime}} & \left(\varepsilon\left|\psi e-\Pi_{h, \varepsilon}(\psi e)\right|_{H^{1}\left(T, \psi^{-1}\right)}^{2}+\varepsilon^{-1}\left\|\psi e-\Pi_{h, \varepsilon}(\psi e)\right\|_{L^{2}\left(T, \psi^{-1}\right)}^{2}\right) \\
\leq & C_{2} \gamma^{-1}\left(\|e\|_{L^{2}\left(\Omega,-D_{\mathbf{c}} \psi\right)}^{2}+\varepsilon|e|_{H^{1}(\Omega, \psi)}^{2}\right) \\
& +C_{2} h^{2 r-1}|u|_{H^{r}\left(\mathcal{N}\left(\Omega^{\prime \prime}\right)\right)}^{2} .
\end{aligned}
$$

and

$$
\begin{aligned}
\sum_{T \in \mathcal{T}_{h}^{\prime \prime}} & \left(\varepsilon\left|\psi e-\Pi_{h, \varepsilon}(\psi e)\right|_{H^{1}(T)}^{2}+\varepsilon^{-1}\left\|\psi e-\Pi_{h, \varepsilon}(\psi e)\right\|_{L^{2}(T)}^{2}\right) \\
\leq & C_{2} \gamma^{-1} h^{2 s}\left(\|e\|_{L^{2}\left(\Omega,-D_{\mathbf{c}} \psi\right)}^{2}+\varepsilon|e|_{H^{1}(\Omega, \psi)}^{2}\right)+C_{2} h^{2 s+2 r-1}|u|_{H^{r}\left(\mathcal{N}\left(\Omega^{\prime \prime}\right)\right)}^{2} \\
& +C_{2} h^{4 s}\left(\|e\|_{L^{2}\left(\mathcal{C}\left(\Omega^{\prime \prime}\right)\right)}^{2}+\varepsilon|e|_{H^{1}\left(\mathcal{C}\left(\Omega^{\prime \prime}\right)\right)}^{2}\right) .
\end{aligned}
$$

Proof. In this proof the constants $C$ only depend on $\Omega^{\prime \prime}, \mathcal{T}, \mathbf{c}, k$ and $s$. Moreover we consider $\gamma$ large enough to guarantee that $h_{T} \leq \gamma h, \forall T \in \mathcal{T}_{h}$, where $h_{T}$ denotes the diameter of $T$, as in (2.1).

Consider first $T \in \mathcal{T}_{h}^{\prime}$. Thanks to (4.6), the weight $\psi$ has bounded oscillations on $T$ :

$$
\max _{\mathbf{x} \in T} \psi(\mathbf{x}) \leq \beta_{2} \min _{\mathbf{x} \in T} \psi(\mathbf{x})
$$


and similarly

$$
\max _{\mathbf{x} \in T} \psi^{-1}(\mathbf{x}) \leq \beta_{2} \min _{\mathbf{x} \in T} \psi^{-1}(\mathbf{x}) .
$$

So we have from (2.15) and (4.20)

$$
\begin{aligned}
& \varepsilon \mid \psi e-\left.\Pi_{h, \varepsilon}(\psi e)\right|_{H^{1}\left(T, \psi^{-1}\right)} ^{2}+\varepsilon^{-1}\left\|\psi e-\Pi_{h, \varepsilon}(\psi e)\right\|_{L^{2}\left(T, \psi^{-1}\right)}^{2} \\
& \leq\left(\max _{\mathbf{x} \in T} \psi^{-1}(\mathbf{x})\right)\left(\varepsilon\left|\psi e-\Pi_{h, \varepsilon}(\psi e)\right|_{H^{1}(T)}^{2}\right. \\
&\left.+\varepsilon^{-1}\left\|\psi e-\Pi_{h, \varepsilon}(\psi e)\right\|_{L^{2}(T)}^{2}\right) \\
& \leq C\left(\min _{\mathbf{x} \in T} \psi^{-1}(\mathbf{x})\right) \sum_{i=1}^{3} h^{2(k+1)}|\psi e|_{H^{k+1}\left(\partial T_{i}\right)}^{2} \\
& \leq C \sum_{i=1}^{3} h^{2(k+1)}|\psi e|_{H^{k+1}\left(\partial T_{i}, \psi^{-1}\right)}^{2} .
\end{aligned}
$$

Now on a single edge $\partial T_{i}$, whose direction is $\mathbf{v}_{i}$, we have

$$
\begin{aligned}
|\psi e|_{H^{k+1}\left(\partial T_{i}, \psi^{-1}\right)}^{2} & =\int_{\partial T_{i}}\left(D_{\mathbf{v}_{i}}^{k+1}(\psi e)\right)^{2} \psi^{-1} d \sigma(\mathbf{x}) \\
& \leq C \int_{\partial T_{i}} \sum_{\substack{m, n \geq 0 \\
m+n=k+1}}\left(D_{\mathbf{v}_{i}}^{m} \psi D_{\mathbf{v}_{i}}^{n} e\right)^{2} \psi^{-1} d \sigma(\mathbf{x}) \\
& \leq C \int_{\partial T_{i}} \sum_{\substack{m, n \geq 0 \\
m+n=k+1}}\left|D_{\mathbf{v}_{i}}^{m} \psi\right|^{2}\left|D_{\mathbf{v}_{i}}^{n} e\right|^{2} \psi^{-1} d \sigma(\mathbf{x}) \\
& \leq C \int_{\partial T_{i}} \sum_{\substack{m, n \geq 0 \\
m+n=k+1}} \underbrace{\sum_{\substack{m_{1}, m_{2} \geq 0 \\
m_{1}+m_{2}=m}}\left|D_{\mathbf{c}}^{m_{1}} D_{\overline{\mathbf{c}}}^{m_{2}} \psi\right|^{2}\left|D_{\mathbf{v}_{i}}^{n} e\right|^{2} d \sigma(\mathbf{x}) .}_{I}
\end{aligned}
$$

In the last integral the addendum with $m=0$ and $n=k+1$ vanishes, because $e$ is a polynomial of order $k$ on each $\partial T_{i}$. For $m \geq 1$ the following estimate holds true:

$$
I \leq-C \gamma^{-1} h^{1-2 m} D_{\mathbf{c}} \psi .
$$

Indeed, using (4.8), (4.11) and (4.15) we get

$$
\begin{aligned}
\psi^{-1}\left|D_{\mathbf{c}} \psi\right|^{2} & \leq \psi^{-1}\left|D_{\mathbf{c}} \psi\right|\left(\beta_{2} \gamma^{-1} h^{-1} \psi+\psi\right) \\
& \leq \beta_{2} \gamma^{-1} h^{-1}(1+\gamma h)\left|D_{\mathbf{c}} \psi\right| \\
& \leq-C \gamma^{-1} h^{-1} D_{\mathbf{c}} \psi ;
\end{aligned}
$$

moreover, when $m_{1}=0$ and hence $m_{2}=m \geq 1$ we have

$$
\begin{aligned}
\psi^{-1}\left|D_{\overline{\mathbf{c}}}^{m} \psi\right|^{2} & \leq C \psi^{-1}\left(\gamma^{-2} h^{-m} \psi^{2}+h^{2-2 m}\left|D_{\mathbf{c}} \psi\right|^{2}+h^{4 s}\right) \\
& \leq C\left(\gamma^{-2} h^{-m} \psi+h^{2-2 m} \psi^{-1}\left|D_{\mathbf{c}} \psi\right|^{2}\right) \\
& \leq C\left(\gamma^{-2} h^{-m}\left|D_{\mathbf{c}} \psi\right|+\gamma^{-1} h^{1-2 m}\left|D_{\mathbf{c}} \psi\right|\right) \\
& \leq-C \gamma^{-1} h^{1-2 m} D_{\mathbf{c}} \psi
\end{aligned}
$$
using (4.5) and (4.23) using (4.11). 
Analogously, in the case $m_{1} \geq 1$ and $m \geq 2$, using (4.5), (4.10), (4.11) and (4.23) we get

$$
\begin{aligned}
\psi^{-1}\left|D_{\mathbf{c}}^{m_{1}} D_{\overline{\mathbf{c}}}^{m_{2}} \psi\right|^{2} & \leq C \psi^{-1} \gamma^{-2} h^{2-2 m}\left|D_{\mathbf{c}} \psi\right|^{2} \\
& \leq-C \gamma^{-1} h^{1-2 m} D_{\mathbf{c}} \psi .
\end{aligned}
$$

Using (4.5), (4.7) and (4.15) we get, as in (4.19)

$$
\max _{\mathbf{x} \in T}\left(-D_{\mathbf{c}} \psi(\mathbf{x})\right) \leq \beta_{2} \min _{\mathbf{x} \in T}\left(-D_{\mathbf{c}} \psi(\mathbf{x})\right),
$$

and then we can apply the estimate (2.27) to the right hand side of (4.21):

$$
\begin{array}{rlrl}
\sum_{i=1}^{3} h^{2(k+1)}|\psi e|_{H^{k+1}\left(\partial T_{i}, \psi^{-1}\right)}^{2} & & \\
\leq & C h \gamma^{-1} \sum_{i=1}^{3} \sum_{n=0}^{k} h^{2 n}|e|_{H^{n}\left(\partial T_{i},-D_{\mathbf{c}} \psi\right)}^{2} & & \text { using (4.22) } \\
\leq & C h \gamma^{-1} \max _{\mathbf{x} \in T}\left(-D_{\mathbf{c}} \psi(\mathbf{x})\right) \sum_{i=1}^{3} \sum_{n=0}^{k} h^{2 n}|e|_{H^{n}\left(\partial T_{i}\right)}^{2} & & \\
\leq & C h \gamma^{-1} \max _{\mathbf{x} \in T}\left(-D_{\mathbf{c}} \psi(\mathbf{x})\right)\|e\|_{L^{2}(\partial T)}^{2} & & \\
\leq & C h \gamma^{-1} \min _{\mathbf{x} \in T}\left(-D_{\mathbf{c}} \psi(\mathbf{x})\right) & & \text { using (2.10) } \\
& \cdot\left(\|e\|_{L^{2}(T)}^{2}+\varepsilon h|e|_{H^{1}(T)}^{2}+h^{2 r}|e|_{H^{r}(\mathcal{N}(T))}^{2}\right) & & \text { using (2.27) } \\
\leq C \gamma^{-1}\left(\|e\|_{L^{2}\left(T,-D_{\mathbf{c}} \psi\right)}^{2}+\varepsilon|e|_{H^{1}(T, \psi)}^{2}\right) & & \text { using (4.8), (4.11), (4.15) } \\
& +C h^{2 r-1}|u|_{H^{r}(\mathcal{N}(T))}^{2} & & \text { using moreover (4.1). }
\end{array}
$$

Summing the previous estimates for all elements $T \in \mathcal{T}_{h}^{\prime}$ and using (2.1) we get (4.17). Now consider $T \in \mathcal{T}_{h}^{\prime \prime}$. In this case

$$
\psi(\mathbf{x}) \leq C h^{2 s}, \quad \forall \mathbf{x} \in T
$$

indeed, if $\psi(\mathbf{x})=\beta_{2} h^{2 s}$ for some $\mathbf{x} \in T$, then (4.6) yields $\psi(\mathbf{x}+\mathbf{y}) \leq \beta_{2} \psi(\mathbf{x}) \leq \beta_{2}^{2} h^{2 s}$, for any $\mathbf{y}$ such that $\mathbf{x}+\mathbf{y} \in T$. In the same way, using (4.7), either

$$
-D_{\mathbf{c}} \psi(\mathbf{x}) \geq \beta_{2} h^{2 s}
$$

or

$$
-D_{\mathbf{c}} \psi(\mathbf{x}) \leq C h^{2 s}
$$

As in the first part we get

$$
\begin{aligned}
\varepsilon \mid \psi e & -\left.\Pi_{h, \varepsilon}(\psi e)\right|_{H^{1}(T)} ^{2}+\varepsilon^{-1}\left\|\psi e-\Pi_{h, \varepsilon}(\psi e)\right\|_{L^{2}(T)}^{2} \\
& \leq C \sum_{i=1}^{3} \int_{\partial T_{i}} \sum_{\substack{m, n \geq 0 \\
m+n=k+1}} \underbrace{\sum_{\substack{m_{1}, m_{2} \geq 0 \\
m_{1}+m_{2}=m}}\left|D_{\mathbf{c}}^{m_{1}} D_{\overline{\mathbf{c}}}^{m_{2}} \psi\right|^{2}\left|D_{\mathbf{v}_{i}}^{n} e\right|^{2} d \sigma(\mathbf{x}) .}_{I I}
\end{aligned}
$$


Assuming (4.26) and $m \geq 1$ we have

$$
I I \leq-C \gamma^{-1} h^{1+2 s-2 m} D_{\mathbf{c}} \psi .
$$

Actually we can proceed as in the first part of the proof, with now the extra assumption (4.25): when $m_{1}=1$ and $m_{2}=0$ we obtain

$$
\begin{aligned}
\left|D_{\mathbf{c}} \psi\right|^{2} & \leq\left|D_{\mathbf{c}} \psi\right|\left(\beta_{2} \gamma^{-1} h^{-1} \psi+h^{2 s}\right) \\
& \leq-C \gamma^{-1} h^{-1+2 s} D_{\mathbf{c}} \psi
\end{aligned}
$$

moreover, when $m_{1}=0 m_{2}=m \geq 1$ we have

$$
\begin{aligned}
\left|D_{\overline{\mathbf{c}}}^{m} \psi\right|^{2} & \leq C\left(\gamma^{-2} h^{-m} \psi^{2}+h^{2-2 m}\left|D_{\mathbf{c}} \psi\right|^{2}+h^{4 s}\right) \\
& \leq-C \gamma^{-1} h^{1+2 s-2 m} D_{\mathbf{c}} \psi,
\end{aligned}
$$

and in the case $m_{1} \geq 1, m \geq 2$ we get

$$
\begin{aligned}
\left|D_{\mathbf{c}}^{m_{1}} D_{\overline{\mathbf{c}}}^{m_{2}} \psi\right|^{2} & \leq C \psi^{-1} \gamma^{-2} h^{2-2 m}\left|D_{\mathbf{c}} \psi\right|^{2} \\
& \leq-C \gamma^{-1} h^{1+2 s-2 m} D_{\mathbf{c}} \psi .
\end{aligned}
$$

Still following the previous analysis we can now conclude:

$$
\begin{aligned}
& \sum_{i=1}^{3} h^{2(k+1)}|\psi e|_{H^{k+1}\left(\partial T_{i}\right)}^{2} \\
& \quad \leq C h^{2 s+1} \gamma^{-1} \sum_{i=1}^{3} \sum_{n=0}^{k} h^{2 n}|e|_{H^{n}\left(\partial T_{i},-D_{\mathbf{c}} \psi\right)}^{2} \\
& \quad \leq C h^{2 s} \gamma^{-1}\left(\|e\|_{L^{2}\left(T,-D_{\mathbf{c}} \psi\right)}^{2}+\varepsilon|e|_{H^{1}(T, \psi)}^{2}\right)+C h^{2 s+2 r-1}|u|_{H^{r}(\mathcal{N}(T))}^{2} .
\end{aligned}
$$

Otherwise if (4.27) hold true from (4.28) we simply obtain the following estimate which does not depend on $\psi$ :

$$
I I \leq C h^{1+4 s-2 m} .
$$

Applying directly (2.10) and (2.27) here we get

$$
\begin{aligned}
& \sum_{i=1}^{3} h^{2(k+1)}|\psi e|_{H^{k+1}\left(\partial T_{i}\right)}^{2} \\
& \quad \leq C h^{4 s+1} \sum_{i=1}^{3} \sum_{n=0}^{k} h^{2 n}|e|_{H^{n}\left(\partial T_{i}\right)}^{2} \\
& \quad \leq C h^{4 s}\left(\|e\|_{L^{2}(T)}^{2}+\varepsilon|e|_{H^{1}(T)}^{2}\right)+C h^{4 s+2 r-1}|u|_{H^{r}(\mathcal{N}(T))}^{2} .
\end{aligned}
$$

In order to get (4.18) we sum the estimates here obtained for any $T \in \mathcal{T}_{h}^{\prime \prime}$ contained in $\mathcal{C}\left(\Omega^{\prime \prime}\right)$, since the triangles outside $\mathcal{C}\left(\Omega^{\prime \prime}\right)$ give no contribution to the left hand side of (4.18).

The following theorem establishes our local error estimate. Actually the error referred to the region $\Omega^{\prime}$ depends also on a global term of order $s$, but we can choose 
$s$ larger than $r-1 / 2$, so that the order of convergence of the method depends only on the local term.

Theorem 4.4. Assume that (1.2), (2.1), (2.2), (2.3) and (2.4) (or (2.5) in the case $k=1)$ are fulfilled; let $s$ be a positive constant and let $\Omega^{\prime}$ and $\Omega^{\prime \prime}$ be open subsets of $\Omega$ with $\Omega^{\prime} \subset \Omega^{\prime \prime}$ and $\Omega^{\prime \prime}$ as in Lemma 4.1. Then there exist constants $C_{1}=$ $C_{1}\left(\Omega^{\prime \prime}, \Omega, \mathcal{T}, s, \mathbf{c}, k\right), C_{2}=C_{2}(\Omega, \mathcal{T}, \mathbf{c}, k)$ and $C_{3}=C_{3}\left(\Omega^{\prime \prime}, \Omega, \mathcal{T}, s, \mathbf{c}, k\right)$ such that, if the distance from $\Omega^{\prime}$ to $\left(\partial \Omega^{\prime \prime}\right)^{0}$ and $\left(\partial \Omega^{\prime \prime}\right)^{+}$is at least $C_{1} h \log h^{-1}$ and $C_{1} h^{1 / 2} \log h^{-1}$ respectively, if $u$ is the solution of (1.4) and $u \in H^{r}\left(\mathcal{N}\left(\Omega^{\prime \prime}\right)\right)$ with $1 \leq r \leq k+1$, if $u_{h}$ is the solution of (1.10) and if $h \leq C_{2}$, then

$$
\begin{gathered}
\varepsilon^{1 / 2}\left|u-u_{h}\right|_{H^{1}\left(\Omega^{\prime}\right)}+h^{-1 / 2}\left\|\mathbf{c} \cdot \nabla\left(u-u_{h}\right)\right\|_{H^{-1}\left(\Omega^{\prime}\right)}+\left\|u-u_{h}\right\|_{L^{2}\left(\Omega^{\prime}\right)} \\
\leq C_{3}\left(h^{r-1 / 2}|u|_{H^{r}\left(\mathcal{N}\left(\Omega^{\prime \prime}\right)\right)}+h^{s}\|f\|_{L^{2}(\Omega)}\right) .
\end{gathered}
$$

Proof. In this proof we assume the constants $C$ and $C_{i}$ to be dependent on $\Omega^{\prime \prime}$, $\Omega, \mathcal{T}, s, \mathbf{c}$ and $k$. In the first part we deal with the coercivity norm. Let $\eta$ be as in definition (2.19). We have from (2.16)

$$
\begin{aligned}
& \varepsilon^{1 / 2}\left|u-u_{h}\right|_{H^{1}\left(\Omega^{\prime}\right)}+\left\|u-u_{h}\right\|_{L^{2}\left(\Omega^{\prime}\right)} \\
& \leq \varepsilon^{1 / 2}|e|_{H^{1}\left(\Omega^{\prime}\right)}+\|e\|_{L^{2}\left(\Omega^{\prime}\right)}+\varepsilon^{1 / 2}|\eta|_{H^{1}\left(\Omega^{\prime}\right)}+\|\eta\|_{L^{2}\left(\Omega^{\prime}\right)} \\
& \leq \varepsilon^{1 / 2}|e|_{H^{1}\left(\Omega^{\prime}\right)}+\|e\|_{L^{2}\left(\Omega^{\prime}\right)}+h^{r-\frac{1}{2}}|u|_{H^{r}\left(\mathcal{N}\left(\Omega^{\prime \prime}\right)\right)} .
\end{aligned}
$$

We use in the sequel the same notation of Lemma 4.1. We consider $\gamma$ large enough, in order that (4.12), (4.17), (4.18) hold true and $\operatorname{diam}(\mathcal{N}(T)) \leq \gamma h, \forall T \in \mathcal{T}_{h}$. Moreover we assume (4.11). Then:

$$
\begin{aligned}
& C\left(\varepsilon|e|_{H^{1}(\Omega, \psi)}^{2}+\|e\|_{L^{2}\left(\Omega,-D_{\mathbf{c}} \psi\right)}^{2}\right) \\
& \quad \leq a(e, \psi e)+h^{2 s}\left(\varepsilon|e|_{H^{1}\left(\Omega^{\prime \prime}\right)}^{2}+\|e\|_{L^{2}\left(\Omega^{\prime \prime}\right)}^{2}\right) \\
& \quad \leq a(e, \psi e)+h^{2 s}\left(\varepsilon|e|_{H^{1}\left(\mathcal{C}\left(\Omega^{\prime \prime}\right)\right)}^{2}+\|e\|_{L^{2}\left(\mathcal{C}\left(\Omega^{\prime \prime}\right)\right)}^{2}\right) \\
& \quad \leq I+I I .
\end{aligned}
$$

Using the Galerkin property (2.20) we have on the first term

$$
\begin{aligned}
I & =a\left(e, \psi e-\Pi_{h, \varepsilon}(\psi e)\right)+a\left(e, \Pi_{h, \varepsilon}(\psi e)\right) \\
& =a\left(e, \psi e-\Pi_{h, \varepsilon}(\psi e)\right)-a\left(\eta, \Pi_{h, \varepsilon}(\psi e)\right) \\
& =a\left(e, \psi e-\Pi_{h, \varepsilon}(\psi e)\right)+a\left(\eta, \psi e-\Pi_{h, \varepsilon}(\psi e)\right)-a(\eta, \psi e) \\
& =I I I+I V+V .
\end{aligned}
$$

We split the term III following Lemma 4.3:

$$
\begin{aligned}
I I I= & \sum_{T \in \mathcal{T}_{h}^{\prime}}\left(\varepsilon \int_{T} \nabla e \cdot \nabla\left(\psi e-\Pi_{h, \varepsilon}(\psi e)\right) d \mathbf{x}+\int_{T} \mathbf{c} \cdot \nabla e\left(\psi e-\Pi_{h, \varepsilon}(\psi e)\right) d \mathbf{x}\right) \\
& +\sum_{T \in \mathcal{T}_{h}^{\prime \prime}}\left(\varepsilon \int_{T} \nabla e \cdot \nabla\left(\psi e-\Pi_{h, \varepsilon}(\psi e)\right) d \mathbf{x}+\int_{T} \mathbf{c} \cdot \nabla e\left(\psi e-\Pi_{h, \varepsilon}(\psi e)\right) d \mathbf{x}\right),
\end{aligned}
$$


and use the Cauchy-Schwarz inequality:

$$
\begin{aligned}
I I I \leq & C \varepsilon^{1 / 2}|e|_{H^{1}(\Omega, \psi)}\left(\sum_{T \in \mathcal{T}_{h}^{\prime}} \varepsilon\left|\psi e-\Pi_{h, \varepsilon}(\psi e)\right|_{H^{1}\left(T, \psi^{-1}\right)}^{2}\right)^{1 / 2} \\
& +C \varepsilon^{1 / 2}|e|_{H^{1}(\Omega, \psi)}\left(\sum_{T \in \mathcal{T}_{h}^{\prime}} \varepsilon^{-1}\left\|\psi e-\Pi_{h, \varepsilon}(\psi e)\right\|_{L^{2}\left(T, \psi^{-1}\right)}^{2}\right)^{1 / 2} \\
& +C \varepsilon^{1 / 2}|e|_{H^{1}\left(\mathcal{C}\left(\Omega^{\prime \prime}\right)\right)}\left(\sum_{T \in \mathcal{T}_{h}^{\prime \prime}} \varepsilon\left|\psi e-\Pi_{h, \varepsilon}(\psi e)\right|_{H^{1}(T)}^{2}\right)^{1 / 2} \\
& +C \varepsilon^{1 / 2}|e|_{H^{1}\left(\mathcal{C}\left(\Omega^{\prime \prime}\right)\right)}\left(\sum_{T \in \mathcal{T}_{h}^{\prime \prime}} \varepsilon^{-1}\left\|\psi e-\Pi_{h, \varepsilon}(\psi e)\right\|_{L^{2}(T)}^{2}\right)^{1 / 2}
\end{aligned}
$$

then (4.17) and (4.18) give

$$
\begin{aligned}
I I I \leq & C\left(\varepsilon^{1 / 2}|e|_{H^{1}(\Omega, \psi)}+h^{s} \varepsilon^{1 / 2}|e|_{H^{1}\left(\mathcal{C}\left(\Omega^{\prime \prime}\right)\right)}\right) \\
\cdot & {\left[C \gamma^{-1 / 2}\left(\|e\|_{L^{2}\left(\Omega,-D_{\mathbf{c}} \psi\right)}+\varepsilon^{1 / 2}|e|_{H^{1}(\Omega, \psi)}\right)+C h^{r-1 / 2}|u|_{H^{r}\left(\mathcal{N}\left(\Omega^{\prime \prime}\right)\right)}\right.} \\
& \left.+C h^{s}\left(\sqrt{\varepsilon}|e|_{H^{1}\left(\mathcal{C}\left(\Omega^{\prime \prime}\right)\right)}+\|e\|_{L^{2}\left(\mathcal{C}\left(\Omega^{\prime \prime}\right)\right)}\right)\right] \\
\leq & C \gamma^{-1 / 2}\left(\varepsilon|e|_{H^{1}(\Omega, \psi)}^{2}+\|e\|_{L^{2}\left(\Omega,-D_{\mathbf{c}} \psi\right)}^{2}\right) \\
+ & C\left(\varepsilon^{1 / 2}|e|_{H^{1}(\Omega, \psi)}+\|e\|_{L^{2}\left(\Omega,-D_{\mathbf{c}} \psi\right)}\right)\left(h^{r-1 / 2}|u|_{H^{r}\left(\mathcal{N}\left(\Omega^{\prime \prime}\right)\right)}+\sqrt{I I}\right) \\
+ & C\left(h^{2 r-1}|u|_{H^{r}\left(\mathcal{N}\left(\Omega^{\prime \prime}\right)\right)}^{2}+I I\right) .
\end{aligned}
$$

In $I V$ we proceed in the same way, and using moreover the error estimate (2.16) for $\eta$, we get

$$
\begin{aligned}
I V \leq & C\left(\varepsilon^{1 / 2}|e|_{H^{1}(\Omega, \psi)}+\|e\|_{L^{2}\left(\Omega,-D_{\mathbf{c}} \psi\right)}\right) \\
& \cdot\left(h^{r-1 / 2}|u|_{H^{r}\left(\mathcal{N}\left(\Omega^{\prime \prime}\right)\right)}+\sqrt{I I}\right) \\
& +C\left(h^{2 r-1}|u|_{H^{r}\left(\mathcal{N}\left(\Omega^{\prime \prime}\right)\right)}^{2}+I I\right) .
\end{aligned}
$$

Moreover

$$
\begin{aligned}
V & \leq \varepsilon \int_{\Omega^{\prime \prime}} e \nabla \eta \cdot \nabla \psi d \mathbf{x}+\varepsilon \int_{\Omega^{\prime \prime}} \psi \nabla \eta \cdot \nabla e d \mathbf{x}+\int_{\Omega^{\prime \prime}} \psi e \mathbf{c} \cdot \nabla \eta d \mathbf{x} \\
& =V I+V I I+V I I I .
\end{aligned}
$$

Using (4.13) we obtain (in the same way as in (4.14) and in the steps following it)

$$
\begin{aligned}
V I & \leq C\left(\varepsilon^{1 / 2}|\eta|_{H^{1}\left(\Omega^{\prime \prime}\right)}\right)\left(\|e\|_{L^{2}\left(\Omega,-D_{\mathbf{c}} \psi\right)}+h^{s}\|e\|_{L^{2}\left(\Omega^{\prime \prime}\right)}\right) \\
& \leq C\left(h^{r-1 / 2}|u|_{H^{r}\left(\mathcal{N}\left(\Omega^{\prime \prime}\right)\right)}\right)\left(\|e\|_{L^{2}\left(\Omega,-D_{\mathbf{c}} \psi\right)}+h^{s}\|e\|_{L^{2}\left(\mathcal{C}\left(\Omega^{\prime \prime}\right)\right)}\right),
\end{aligned}
$$


and

$$
\begin{aligned}
V I I & \leq C\left(\varepsilon^{1 / 2}|\eta|_{H^{1}\left(\Omega^{\prime \prime}\right)}\right)\left(\varepsilon^{1 / 2}|e|_{H^{1}(\Omega, \psi)}\right) \\
& \leq C\left(h^{r-1 / 2}|u|_{H^{r}\left(\mathcal{N}\left(\Omega^{\prime \prime}\right)\right)}\right)\left(\varepsilon^{1 / 2}|e|_{H^{1}(\Omega, \psi)}\right),
\end{aligned}
$$

and finally

$$
\begin{aligned}
V I I I & =-\int_{\Omega} \eta e \mathbf{c} \cdot \nabla \psi d \mathbf{x}-\int_{\Omega} \psi \eta \mathbf{c} \cdot \nabla e d \mathbf{x}-\int_{\Omega} \operatorname{div}(\mathbf{c}) \eta e \psi d \mathbf{x} \\
& \leq C\left(\varepsilon^{-1 / 2}\|\eta\|_{L^{2}\left(\Omega^{\prime \prime}\right)}\right)\left(\|e\|_{L^{2}\left(\Omega,-D_{\mathbf{c}} \psi\right)}+\varepsilon^{1 / 2}|e|_{H^{1}(\Omega, \psi)}\right) \\
& \leq C\left(h^{r-1 / 2}|u|_{H^{r}\left(\mathcal{N}\left(\Omega^{\prime \prime}\right)\right)}\right)\left(\|e\|_{L^{2}\left(\Omega,-D_{\mathbf{c}} \psi\right)}+\varepsilon^{1 / 2}|e|_{H^{1}(\Omega, \psi)}\right) .
\end{aligned}
$$

We take $h$ small enough (as stated by Theorem 3.3) to make the discrete stability condition (3.10) hold true; note that this choice is independent of $\Omega^{\prime \prime}$ and $s$. Then, using (3.10), (1.5), (2.16) and the triangle inequality we get

$$
\begin{aligned}
\varepsilon|e|_{H^{1}\left(\mathcal{C}\left(\Omega^{\prime \prime}\right)\right)}^{2}+\|e\|_{L^{2}\left(\mathcal{C}\left(\Omega^{\prime \prime}\right)\right) \leq}^{2} \leq & |\eta|_{H^{1}\left(\mathcal{C}\left(\Omega^{\prime \prime}\right)\right)}^{2}+\|\eta\|_{L^{2}\left(\mathcal{C}\left(\Omega^{\prime \prime}\right)\right)}^{2} \\
& +\varepsilon|u|_{H^{1}\left(\mathcal{C}\left(\Omega^{\prime \prime}\right)\right)}^{2}+\|u\|_{L^{2}\left(\mathcal{C}\left(\Omega^{\prime \prime}\right)\right)}^{2} \\
& +\varepsilon\left|u_{h}\right|_{H^{1}\left(\mathcal{C}\left(\Omega^{\prime \prime}\right)\right)}^{2}+\left\|u_{h}\right\|_{L^{2}\left(\mathcal{C}\left(\Omega^{\prime \prime}\right)\right)}^{2} \\
\leq & C\left(\|f\|_{L^{2}(\Omega)}^{2}+h^{2 r-1}|u|_{H^{r}\left(\mathcal{N}\left(\Omega^{\prime \prime}\right)\right)}^{2}\right),
\end{aligned}
$$

so

$$
I I \leq C h^{2 s}\left(\|f\|_{L^{2}(\Omega)}^{2}+h^{2 r-1}|u|_{H^{r}\left(\mathcal{N}\left(\Omega^{\prime \prime}\right)\right)}^{2}\right) .
$$

Returning to (4.31) we finally get

$$
\begin{aligned}
\left(C_{1}-\right. & \left.C_{2} \gamma^{-1 / 2}\right)\left(\varepsilon|e|_{H^{1}(\Omega, \psi)}^{2}+\|e\|_{L^{2}\left(\Omega,-D_{\mathbf{c}} \psi\right)}^{2}\right) \\
\leq & C\left(h^{r-1 / 2}|u|_{H^{r}\left(\mathcal{N}\left(\Omega^{\prime \prime}\right)\right)}+h^{s}\|f\|_{L^{2}(\Omega)}\right) \\
& \cdot\left(\varepsilon^{1 / 2}|e|_{H^{1}(\Omega, \psi)}+\|e\|_{L^{2}\left(\Omega,-D_{\mathbf{c}} \psi\right)}\right) \\
& +C h^{2 s}\left(h^{2 r-1}|u|_{H^{r}\left(\mathcal{N}\left(\Omega^{\prime \prime}\right)\right)}^{2}+\|f\|_{L^{2}(\Omega)}^{2}\right)
\end{aligned}
$$

hence, choosing $\gamma$ large enough, we get

$$
\varepsilon^{1 / 2}|e|_{H^{1}(\Omega, \psi)}+\|e\|_{L^{2}\left(\Omega,-D_{\mathbf{c}} \psi\right)} \leq C\left(h^{r-1 / 2}|u|_{H^{r}\left(\mathcal{N}\left(\Omega^{\prime \prime}\right)\right)}+h^{s}\|f\|_{L^{2}(\Omega)}\right) .
$$

Collecting (4.30) and (4.32), from (4.3), we get the error bound without the streamline derivative term.

Now our analysis is devoted to the streamline derivative part of the error. Let $v$ be a function in $H_{0}^{1}\left(\Omega^{\prime}\right)$. As in Theorem 2.5, with $\mathcal{N}\left(\Omega^{\prime}\right)$ instead of $\Omega$, we have

$$
\begin{aligned}
\int_{\Omega^{\prime}} \mathbf{c} \cdot \nabla\left(u-u_{h}\right) v d \mathbf{x}= & \int_{\mathcal{N}\left(\Omega^{\prime}\right)} \mathbf{c} \cdot \nabla\left(u-u_{h}\right)\left(v-P_{h, \varepsilon}(v)\right) d \mathbf{x} \\
& +\int_{\mathcal{N}\left(\Omega^{\prime}\right)} \mathbf{c} \cdot \nabla\left(u-u_{h}\right) P_{h, \varepsilon}(v) d \mathbf{x} \\
\leq & C\left(\varepsilon^{1 / 2}\left|u-u_{h}\right|_{H^{1}\left(\mathcal{N}\left(\Omega^{\prime}\right)\right)}\right)\left(h^{1 / 2}|v|_{H^{1}\left(\mathcal{N}\left(\Omega^{\prime}\right)\right)}\right) .
\end{aligned}
$$


Note that for any $\mathbf{x} \in \mathcal{N}\left(\Omega^{\prime}\right)$ there exists a point $\mathbf{y} \in \Omega^{\prime}$, with $|\mathbf{y}| \leq \gamma h$. Applying (4.3) and (4.6) we have $1 \leq \psi(\mathbf{x}+\mathbf{y}) \leq \beta_{2} \psi(\mathbf{x})$. In other words

$$
\psi(\mathbf{x}) \geq \beta_{2}^{-1}, \quad \forall \mathbf{x} \in \mathcal{N}\left(\Omega^{\prime}\right) .
$$

Then by the first part of our proof

$$
\begin{aligned}
\varepsilon^{1 / 2}\left|u-u_{h}\right|_{H^{1}\left(\mathcal{N}\left(\Omega^{\prime}\right)\right)} & \leq C\left(\varepsilon^{1 / 2}|e|_{H^{1}(\Omega, \psi)}+\varepsilon^{1 / 2}|\eta|_{H^{1}\left(\mathcal{N}\left(\Omega^{\prime}\right)\right)}\right) \\
& \leq C\left(h^{r-1 / 2}|u|_{H^{r}\left(\mathcal{N}\left(\Omega^{\prime \prime}\right)\right)}+h^{s}\|f\|_{L^{2}(\Omega)}\right)
\end{aligned}
$$

as $v$ is assumed to be zero on $\mathcal{N}\left(\Omega^{\prime}\right) \backslash \Omega^{\prime}$

$$
|v|_{H^{1}\left(\mathcal{N}\left(\Omega^{\prime}\right)\right)}=|v|_{H^{1}\left(\Omega^{\prime}\right)}
$$

and then we obtain

$$
\int_{\Omega^{\prime}} \mathbf{c} \cdot \nabla\left(u-u_{h}\right) v d \mathbf{x} \leq C h^{1 / 2}\left(h^{r-1 / 2}|u|_{H^{r}\left(\mathcal{N}\left(\Omega^{\prime \prime}\right)\right)}+h^{s}\|f\|_{L^{2}(\Omega)}\right) \cdot|v|_{H^{1}\left(\Omega^{\prime}\right)},
$$

that is

$$
h^{-1 / 2}\left\|\mathbf{c} \cdot \nabla\left(u-u_{h}\right)\right\|_{H^{-1}\left(\Omega^{\prime}\right)} \leq C\left(h^{r-\frac{1}{2}}|u|_{H^{r}\left(\mathcal{N}\left(\Omega^{\prime \prime}\right)\right)}+h^{s}\|f\|_{L^{2}(\Omega)}\right),
$$

and the proof is concluded.

5. Extensions and conclusion. Our analysis is developed for 2-D problems: this simplifies the notation and some of our proofs. Actually the same analysis could be carried out without modifications for $N$-D problems (i.e. when $\Omega$ is a subset of $\mathbb{R}^{N}$, the other hypotheses being adjusted accordingly) when $N \leq 4$, while for $N>4$ we need a different definition for the operator $\Pi_{h, \varepsilon}$. The case $N=1$ is trivial because the continuous formulation and the RFB formulation are equivalent (see [8]).

The results here obtained need the restrictive hypothesis (2.4) for the case $k \geq 2$, i.e. the assumption that all edges of triangulation are bounded away from the direction of the flow. It remains to investigate the possibility of the same results being valid under weaker assumptions.

\section{REFERENCES}

[1] S. M. Alessandrini, D. N. Arnold, R. S. Falk and A. L. Madureira, Derivation and justification of plate models by variational methods, in Plates And Shells, M. Fortin, ed., CRM Proceeding and Lecture Notes, vol. 21, AMS, Providence, RI, 1999, pp. 1-20.

[2] C. Baiocchi and F. Brezzi, Stabilization of unstable numerical methods, in Current Problems Of Analysis And Mathematical Physics (Italian), Univ. Roma "La Sapienza", Rome, 1993, pp. 59-63.

[3] C. Baiocchi, F. Brezzi and L. P. Franca, Virtual bubbles and Galerkin-least-squares type methods (Ga.L.S.), Comp. Meth. Appl. Mech. Engrg., 105 (1993), pp. 125-141.

[4] F. Brezzi, L. P. Franca, T. J. R. Hughes and A. Russo, $b=\int g$, Comp. Meth. Appl. Mech. Engrg., 145 (1997), pp. 329-339.

[5] F. Brezzi, L. P. Franca and A. Russo, Further considerations on residual free bubbles for advective-diffusive equations, Comp. Meth. Appl. Mech. Engrg., 166 (1998), pp. 25-33.

[6] F. BREzZI, D. MARINI AND E. SüLI, Residual-free bubbles for advection-diffusion problems: the general error analysis, Numer. Math., 85 (2000), pp. 31-37.

[7] F. Brezzi, T. J. R. Hughes, D. Marini, A. Russo And E. Süli, A priori error analysis of a finite element method with residual-free bubbles for advection-dominated equations, SIAM J. Numer. Anal., 36 (1999), pp. 1933-1948. 
[8] F. Brezzi And A. Russo, Choosing bubbles for advection-diffusion problems, Math. Models Methods Appl. Sci., 4 (1994), pp. 571-587.

[9] A. N. Brookes And T. J. R. Hughes, Streamline upwind/Petrov-Galerkin formulations for convection dominated flows with particular emphasis on the incompressible Navier-Stokes equations, Comp. Meth. Appl. Mech. Engrg., 82 (1982), pp.199-259.

[10] P. G. Ciarlet, The Finite Element Method for Elliptic Problems, North-Holland, Amsterdam, 1978.

[11] G. Duvaut and J. L. Lions, Les Inéquation en Mécanique et en Physique, Dunod, Paris, 1976.

[12] L. P. Franca, S. L. Frey and T. J. R. Hughes, Stabilized finite element methods: I. Application to the advective-diffusive model, Comp. Meth. Appl. Mech. Engrg., 95 (1992), pp. 253-276.

[13] L. P. Franca, A. Nesliturk and M. Stynes, On the stability of residual-free bubbles for convection-diffusion problems and their approximation by a two-level finite element method, Comp. Meth. Appl. Mech. Engrg., 166 (1998), pp. 35-49.

[14] L. P. Franca And A. Russo, A formulation based on residual-free bubbles for a singular perturbation equation, in preparation.

[15] H. Goering, A. Felgenhauer, G. Lube, H.-G. Roos, L. Tobiska, Singularly Perturbed Differential Equations, Akademie-Verlag, Berlin, 1983.

[16] P. Grisvard, Elliptic Problems In Nonsmooth Domains, Pitman, London, 1985.

[17] C. JohNSON AND U. NäverT An analysis of some finite elements nethods for advection diffusion problems, in Analytical and Numerical Approaches to Asymptotic Problems in Analysis, O. Axelsson, L.S. Frank, and A. Van Der Sluis, eds., North-Holland, Amsterdam, 1981, pp. $99-116$.

[18] C. Johnson, A. H. Schatz And L. B. Wahlbin, Crosswind smear and pointwise errors in streamline diffusion finite element methods, Math. Comp. 49 (1987), pp. 25-38.

[19] U. NÄverT, A Finite Element Method For Convection-Diffusion Problems, Ph.D. thesis at Chalmers University of Technology, Göteborg, 1982.

[20] H.-G. Roos, M. Stynes And L. Tobiska, Numerical Methods for Singularly Perturbed Differential Equations, Springer-Verlag, Berlin, 1996.

[21] R. VerfürTh, Error estimates for some quasi-interpolation operators, Technical report. Department of Mathematics, Univerität Bochum (1997).

[22] G. ZHOU, How accurate is the streamline diffusion finite element method?, Math. Comp. 66 (1997), pp. 31-44.

[23] G. Zhou and R. Rannacher, Pointwise superconvergence of streamline diffusion finite element method, Numer. Methods Partial Differential Equations 12 (1996), pp. 123-145. 